\title{
The Timepix3 Telescope and Sensor R\&D for the LHCb VELO Upgrade
}

\author{
Elena Dall'Occo \\ on behalf of the LHCb VELO Upgrade Group
}

IEEE NSS/MIC 2018

Sydney $10-17 / 11 / 2018$ 


\section{VELO Upgrade}

\section{LHCb Detector \\ (Upgrade 2019/2020)}

Luminosity:

$4 \times 10^{32} \mathrm{~cm}^{-2} \mathrm{~s}^{-1} \stackrel{\times 5}{\longrightarrow} 2 \times 10^{33} \mathrm{~cm}^{-2} \mathrm{~s}^{-1}$

VErtex LOcator

from strip sensors to hybrid pixel detectors
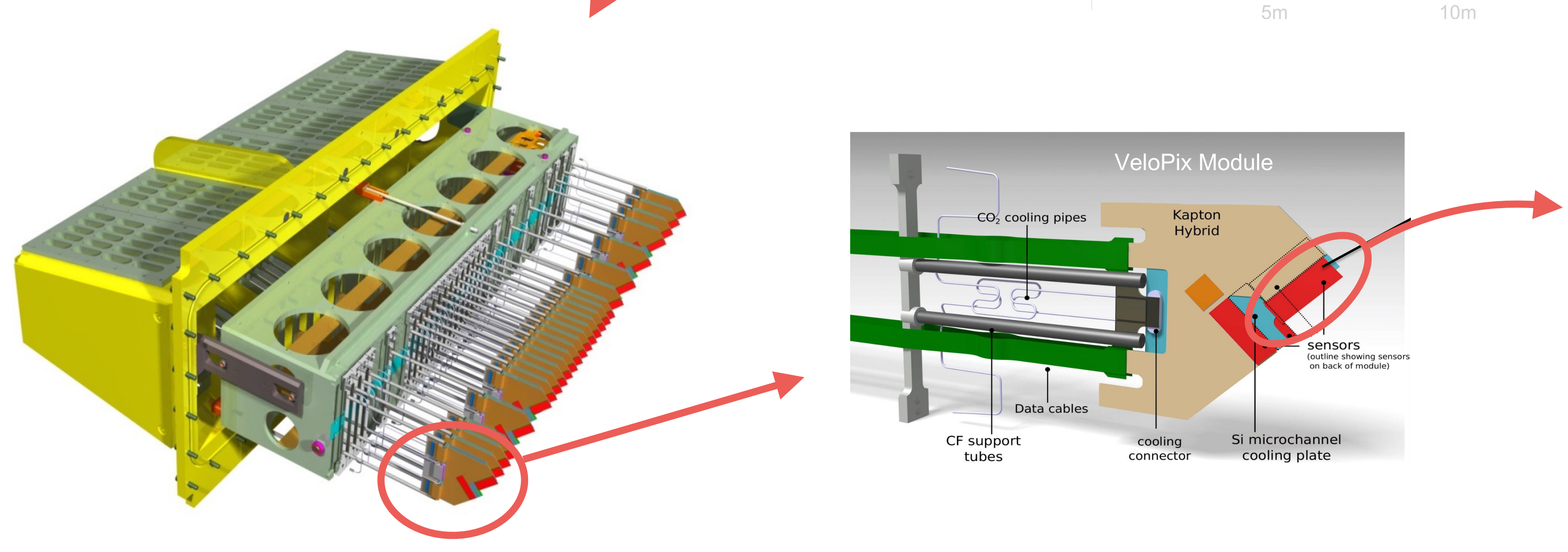

VELO Upgrade

- Prototype Sensors

- Timepix3 Telescope

- Time Resolution

- Pointing Resolution

- Rate Performance

- Sensor Characterisation

- HV Tolerance

Charge Collection

Efficiency

Spatial Resolution

Edge

Grazing Angles

- summary

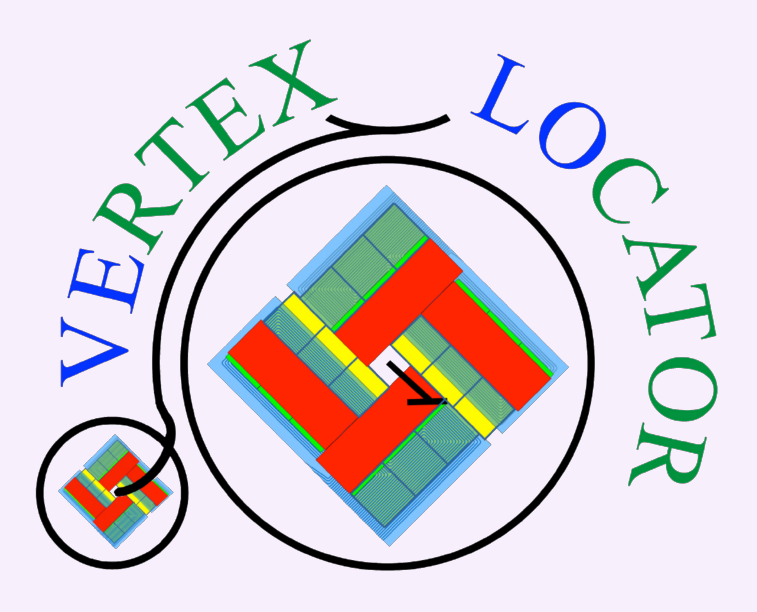




\section{Challenges}

- Increased data rate and fluence

- Non uniform radiation exposure:

- $8 \times 10151 \mathrm{MeV} \mathrm{n}_{\text {eq }} / \mathrm{cm}^{2}$ at the close edge

$0.2 \times 10^{15} 1 \mathrm{MeV} \mathrm{neq}_{\mathrm{e}} / \mathrm{cm}^{2}$ at the outer edge

- Minimise material in the acceptance

\section{Requirements}

- Sensor must withstand 1000V at the end of lifetime

- Charge collection $>6000 e^{-}$

- Efficiency $>99 \%$ and uniform

- Excellent spatial resolution

- Narrow edge for impact parameter resolution

minimise material before the first measured point

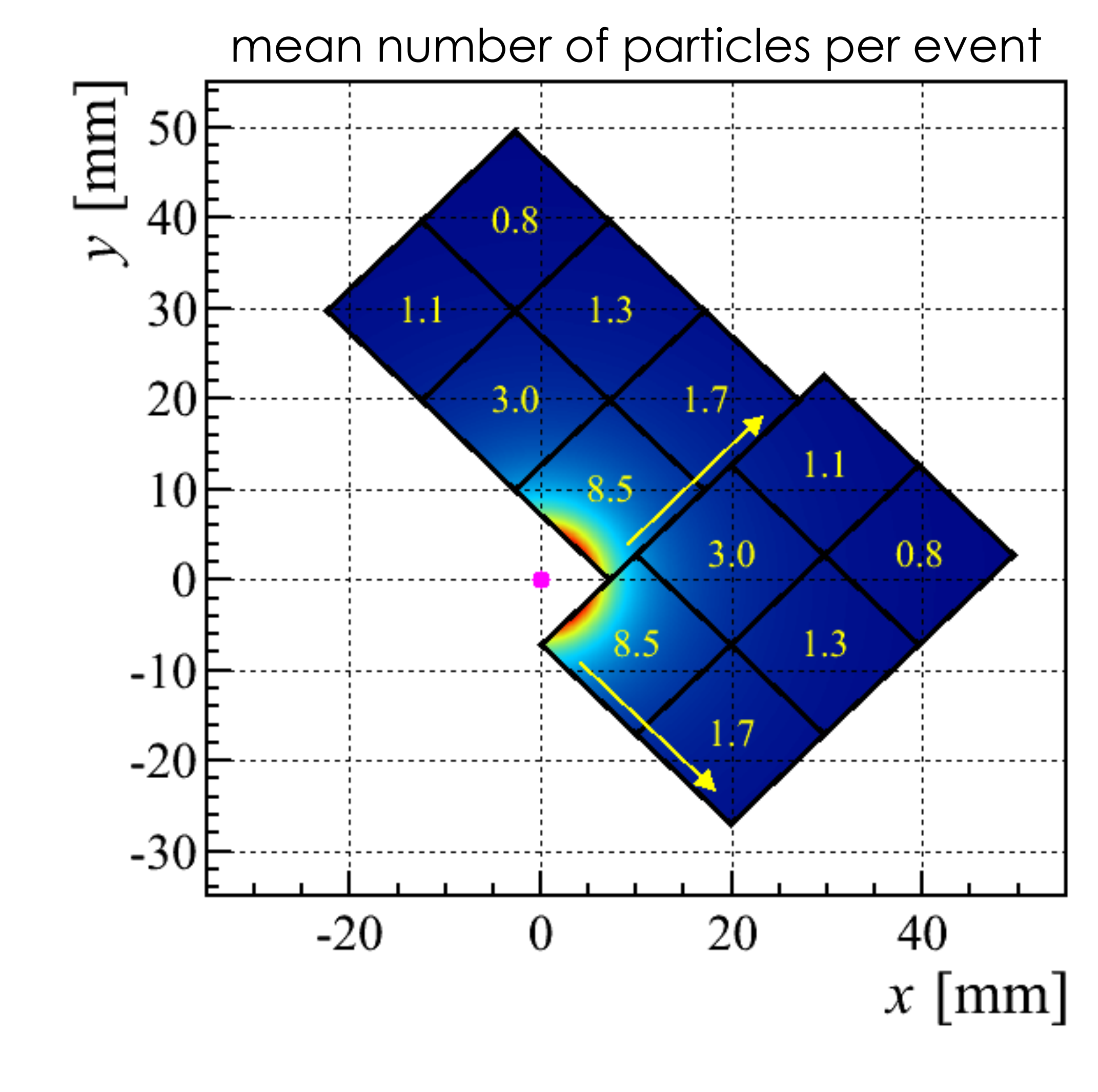

VELO Upgrade

- Prototype Sensors

- Timepix3 Telescope

- Time Resolution

- Pointing Resolution

Rate Performance

- Sensor Characterisation

- HV Tolerance

Charge Collection

- Efficiency

Spatial Resolution

Edge

Grazing Angles

- Summary

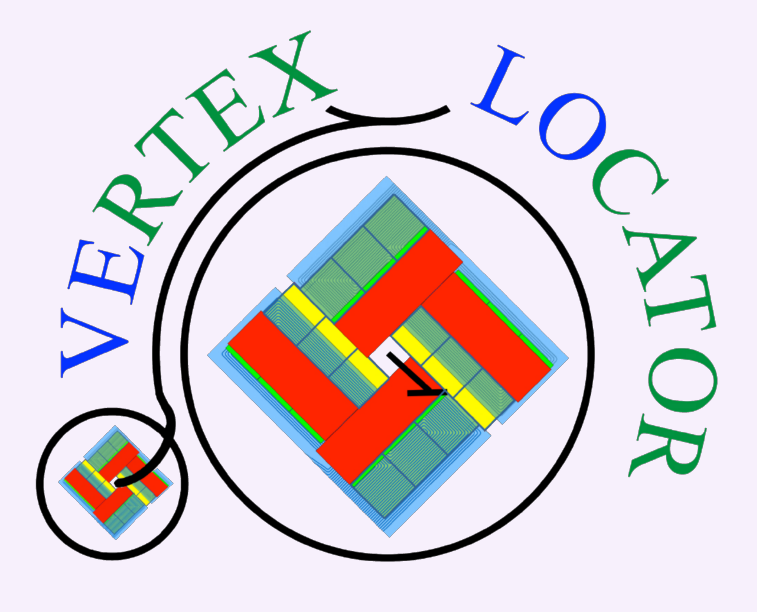


- two different vendors (HPK and Micron)

- n-on-p and n-on-n type

- different thickness (150-200 $\mu \mathrm{m})$

- different implant width (35-39 $\mu \mathrm{m})$

- different guard ring size (150-600 $\mu \mathrm{m})$

- irradiated up to a fluence of $8 \times 10^{15} 1 \mathrm{MeV} \mathrm{n}_{\text {eq }} / \mathrm{cm}^{2}$ (uniformly and non-uniformly irradiated)

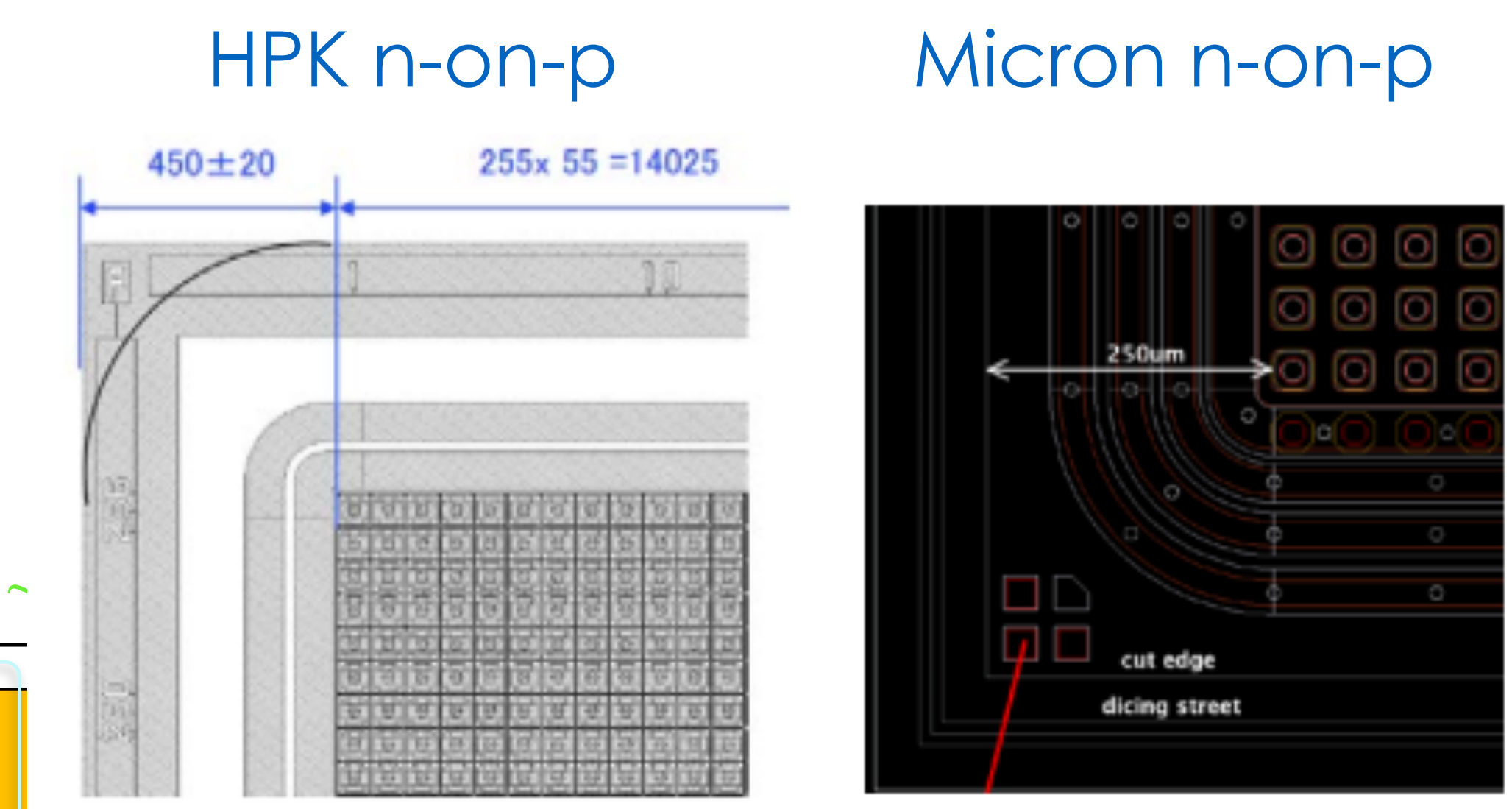

\section{Sensors tested in combination with Timepix 3 ASIC}

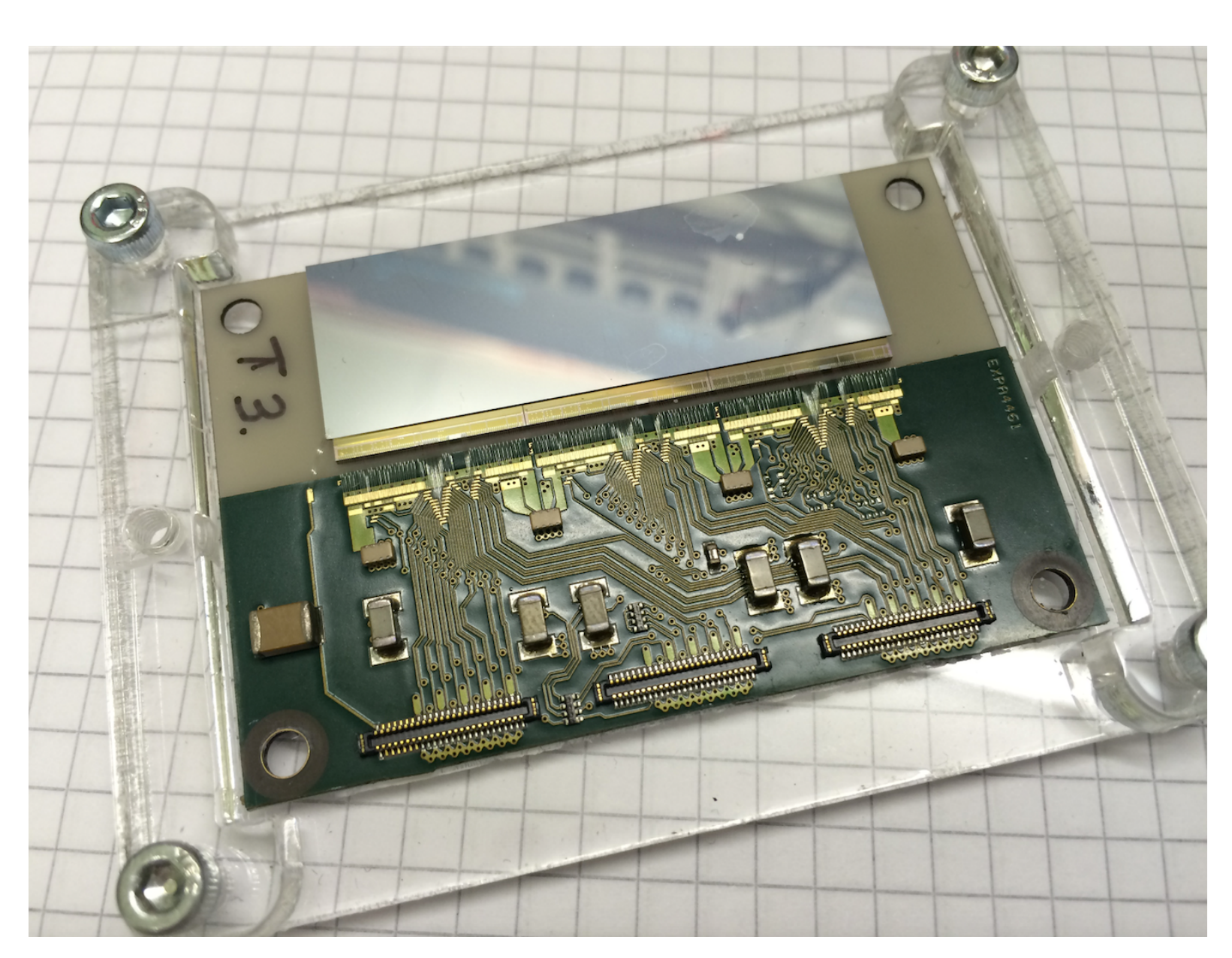

Timepix3

- matrix 256x256 square pixels

- pixel size 55x55 $\mathrm{mm}^{2}$

- can measure simultaneously ToA and ToT (can be converted to charge)

- gives a timestamp with 1.6 ns resolution (bin size of the TDC)

- calibration with test pulse, cross checked with radioactive sources
Micron n-on-n

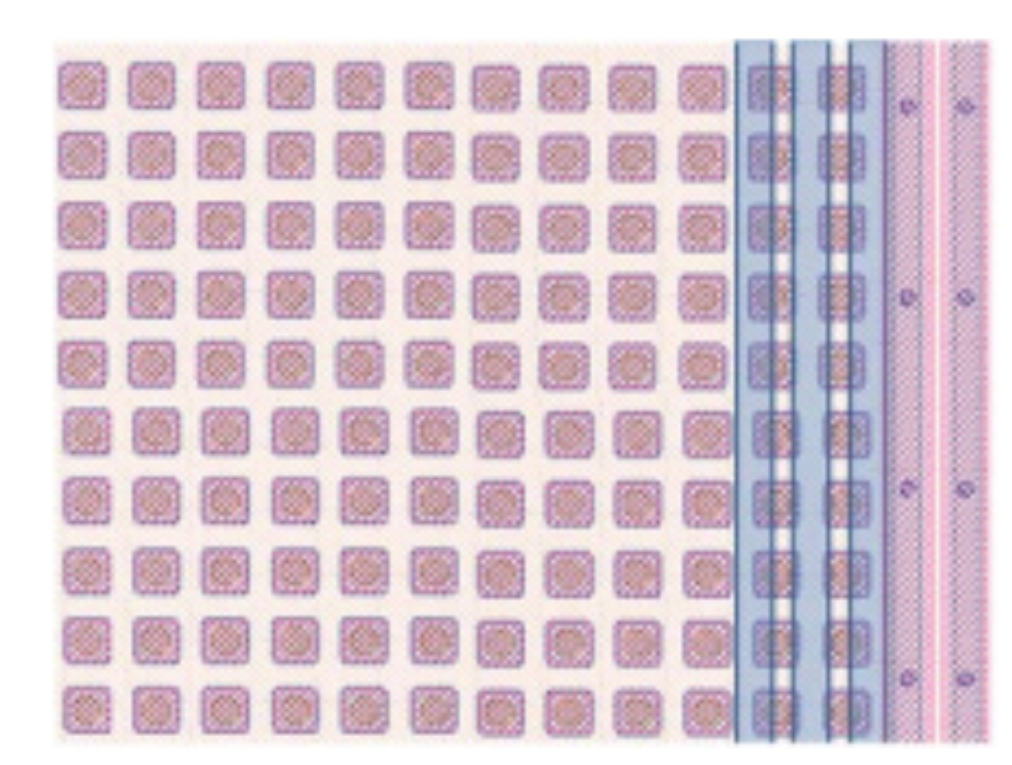

- VELO Upgrade

- Prototype Sensors

- Timepix3 Telescope

- Time Resolution

- Pointing Resolution

Rate Performance

- Sensor Characterisation

- HV Tolerance

- Charge Collection

- Efficiency

- Spatial Resolution

- Edge

- Grazing Angles

- Summary

ideal for sensor testing!

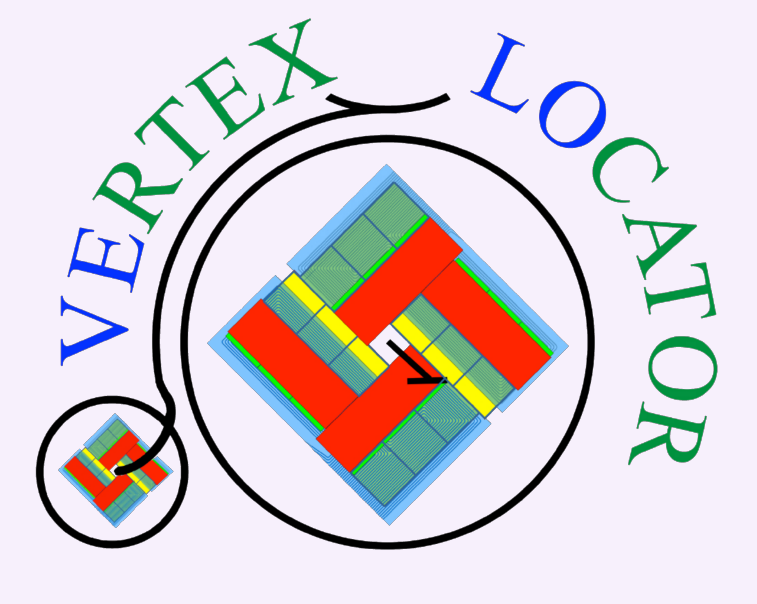




\section{Timepix3 Telescope}

\section{- Operated on a $180 \mathrm{GeV}$ hadron beam at the CERN SPS}

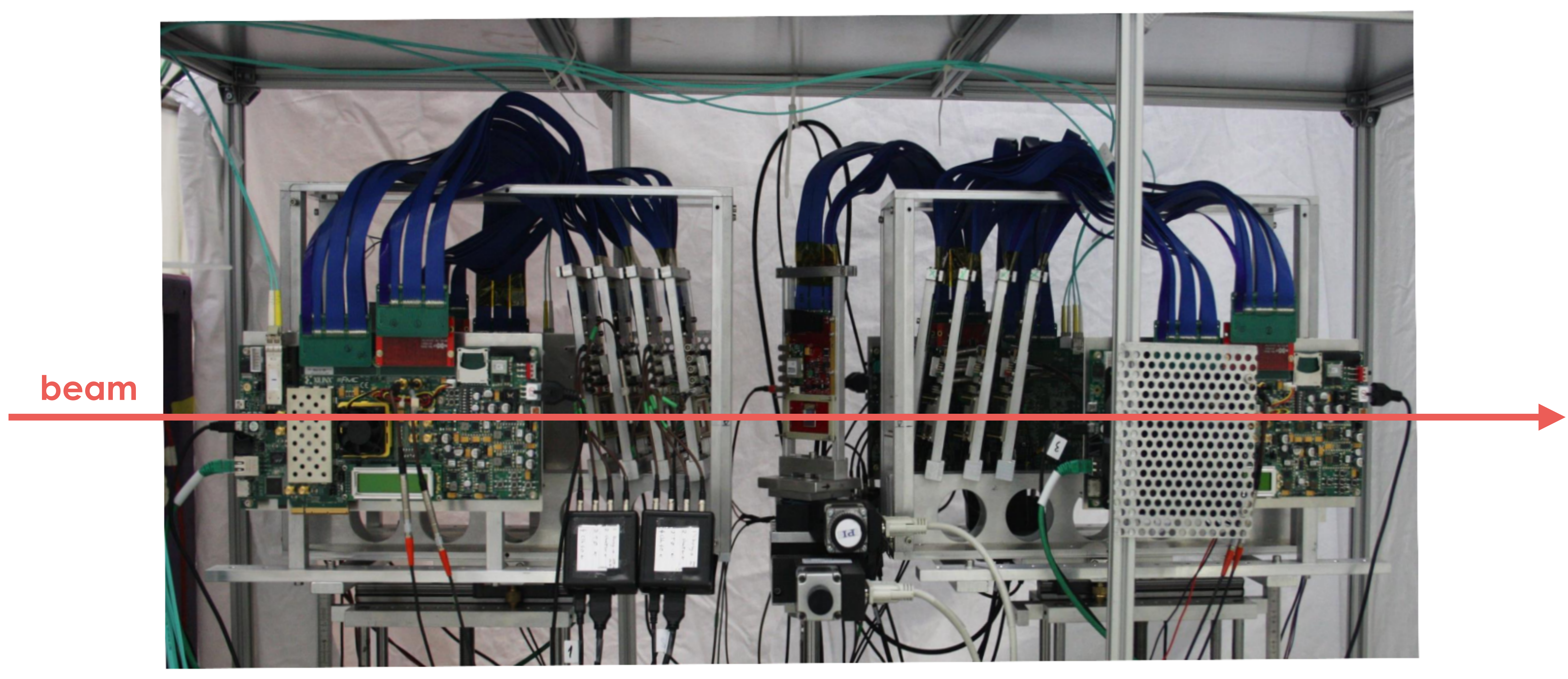

- VELO Upgrade

- Prototype Sensors

- Timepix3 Telescope

- Time Resolution

Pointing Resolution

Rate Performance

- Sensor Characterisation

- HV Tolerance

- Charge Collection

Efficiency

Spatial Resolution

Edge

Grazing Angles

- Summary

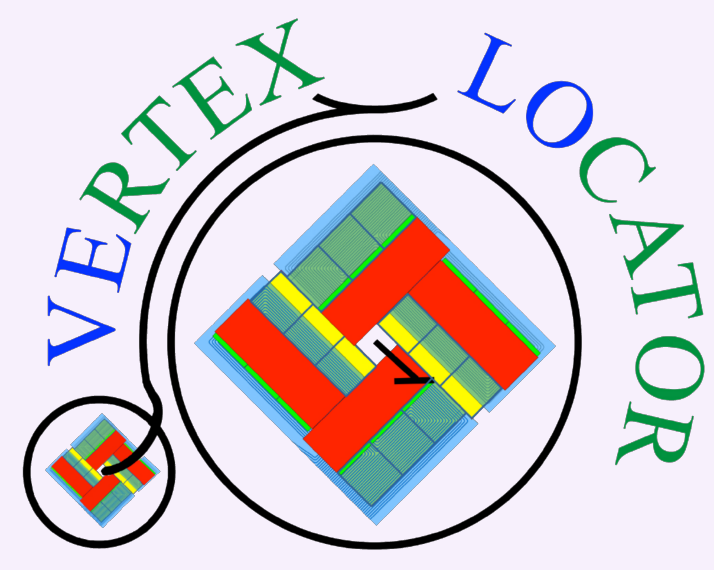




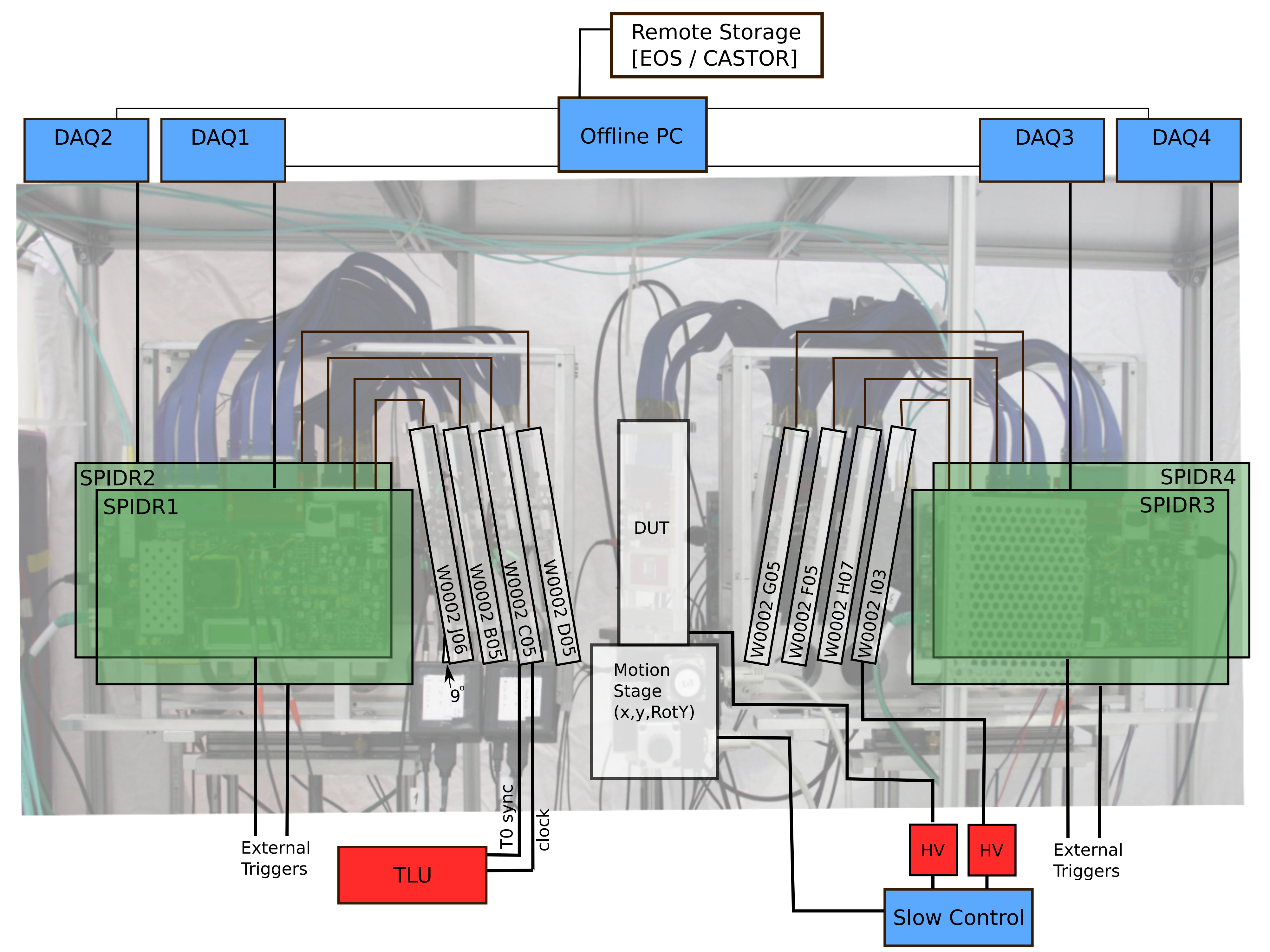

- VELO Upgrade

- Prototype Sensors

- Timepix3 Telescope

- Time Resolution

- Pointing Resolution

- Rate Performance

- Sensor Characterisation

- HV Tolerance

- Charge Collection

- Efficiency

- Spatial Resolution

- Edge

- Grazing Angles

- Summary

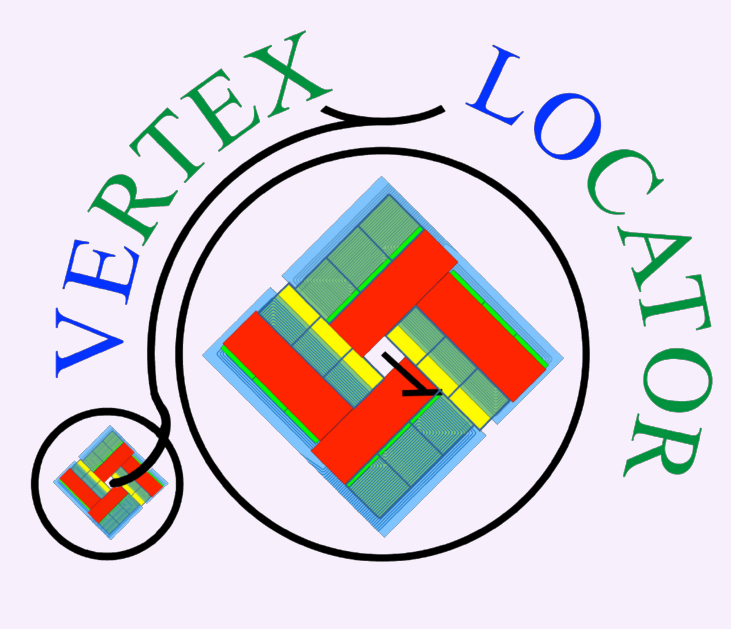


- Telescope planes are time aligned

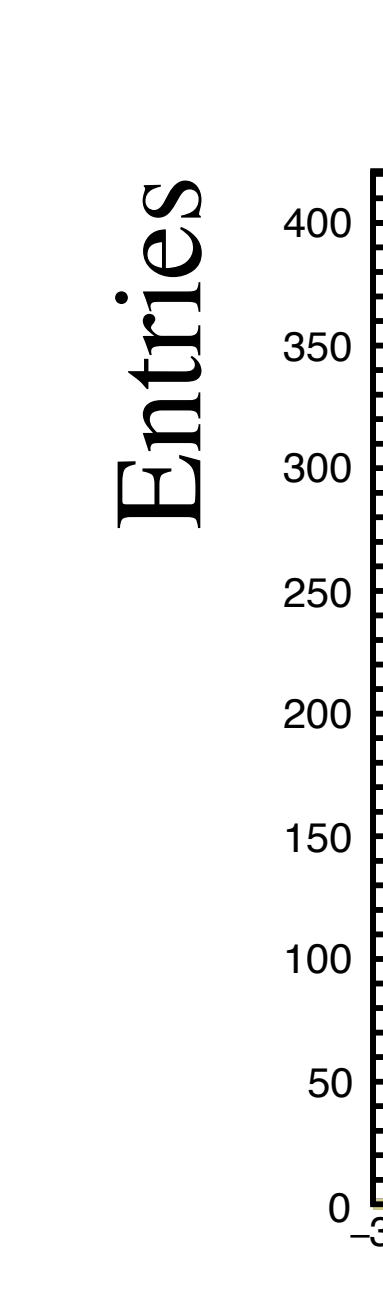

Mean of the biased track time residuals

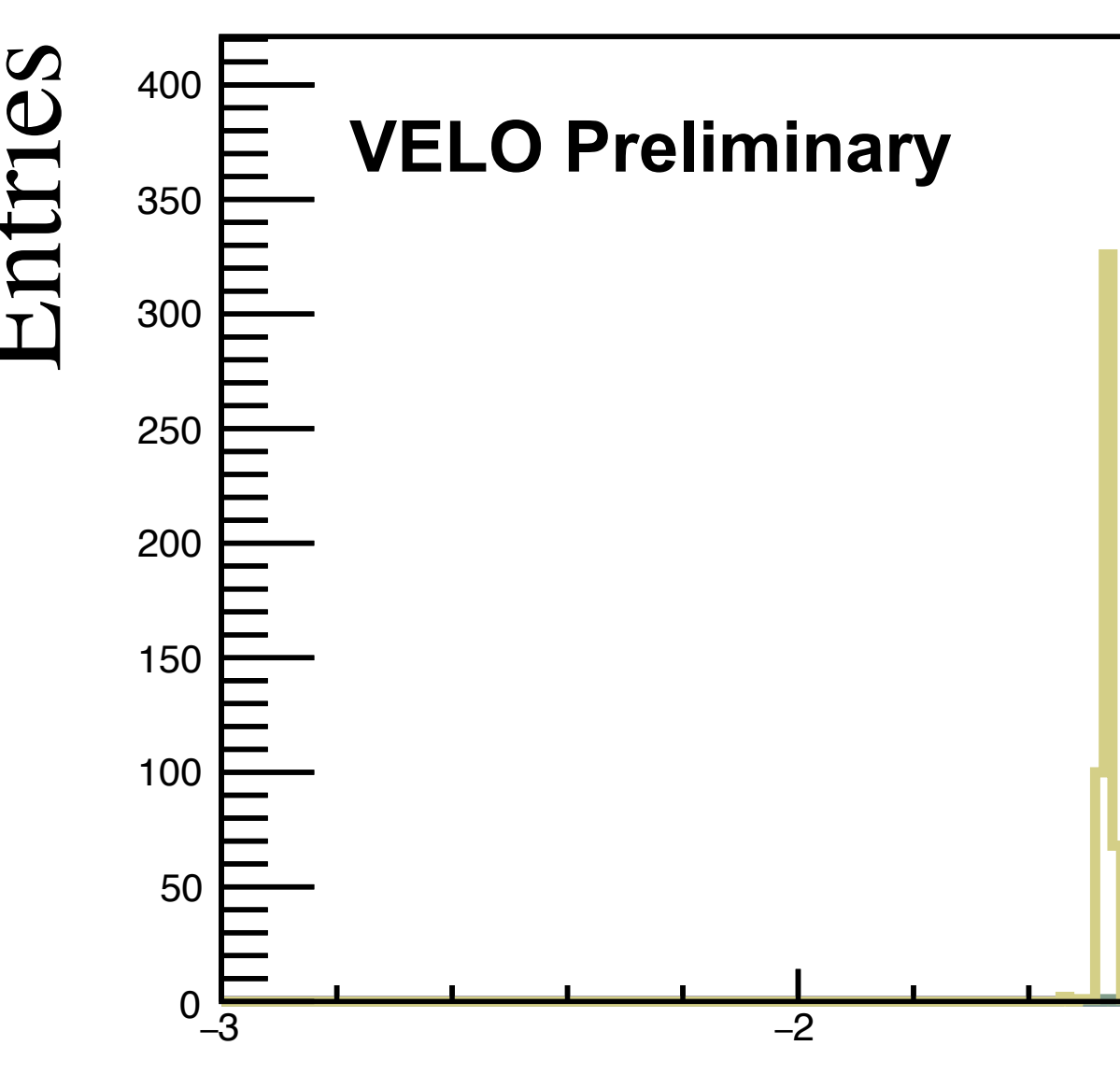

Biased time residuals for one

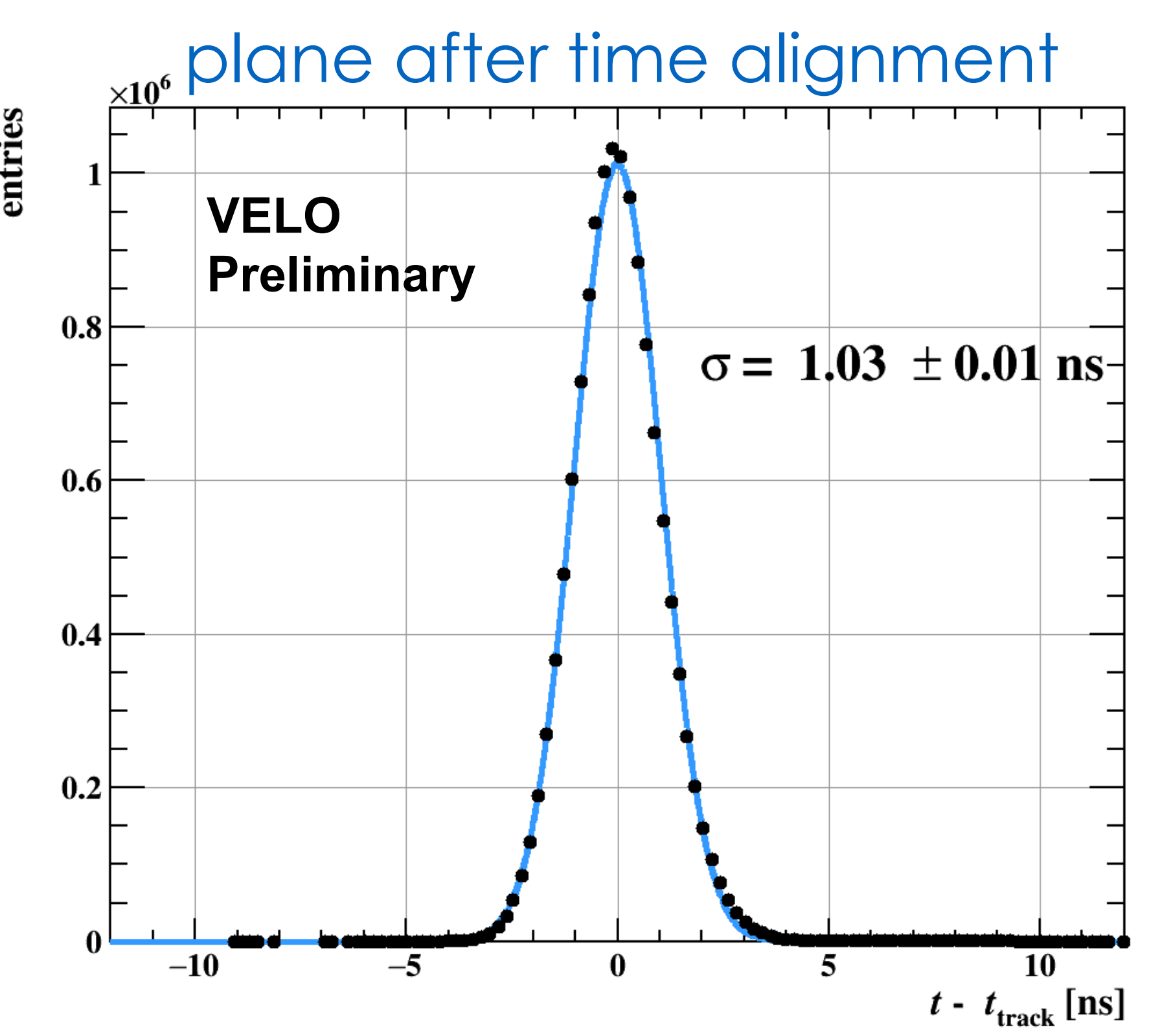

- Telescope time resolution:

$\sigma_{\text {telescope }}=\sigma_{\text {intrinsic }} / \sqrt{ } \mathrm{N}_{\text {planes }}$

Telescope time resolution 0.39 ns at $150 \mathrm{~V}$ bias voltage

Measured intrinsic resolution of each plane $\sim 1.1 \mathrm{~ns}$
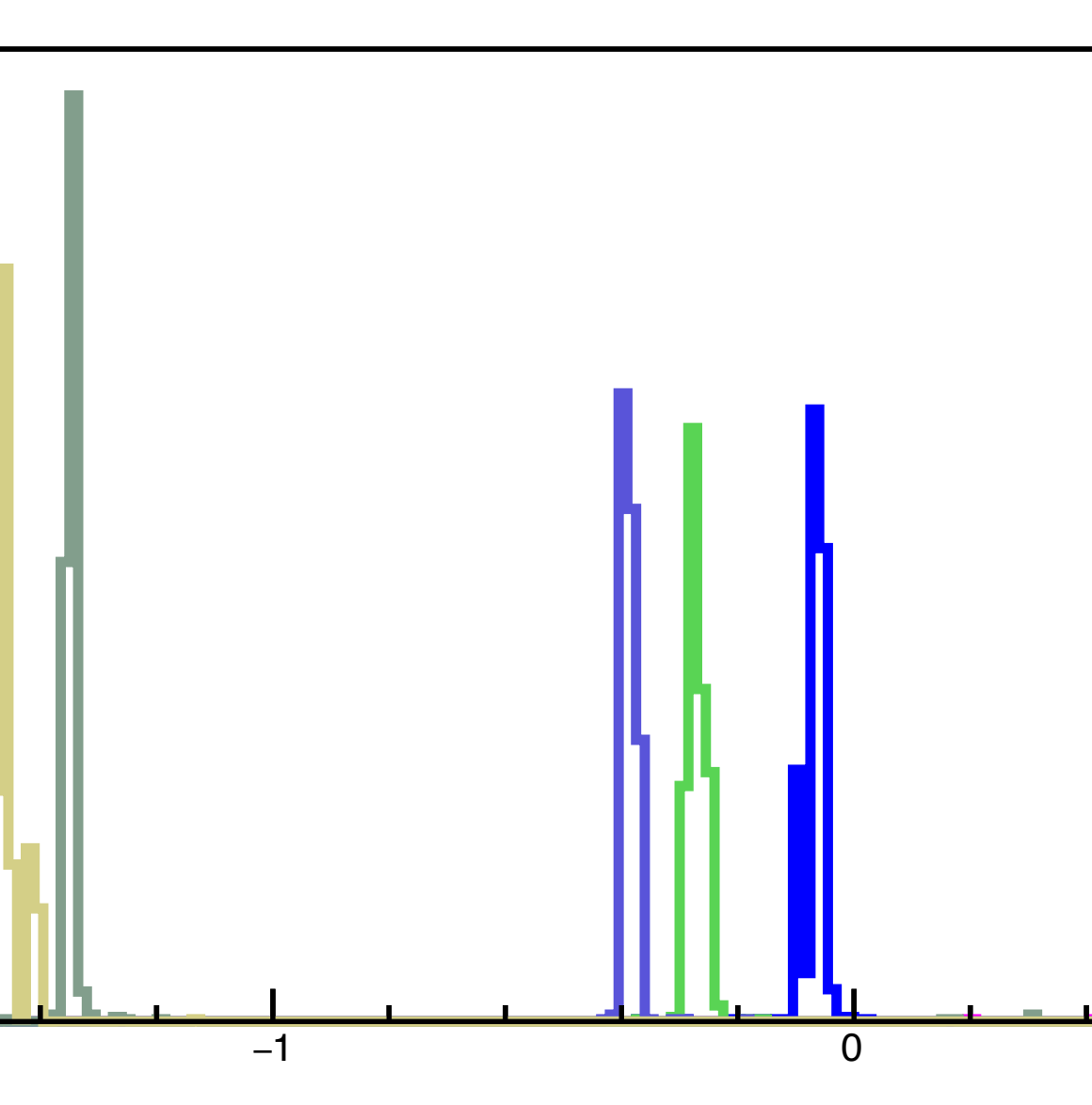

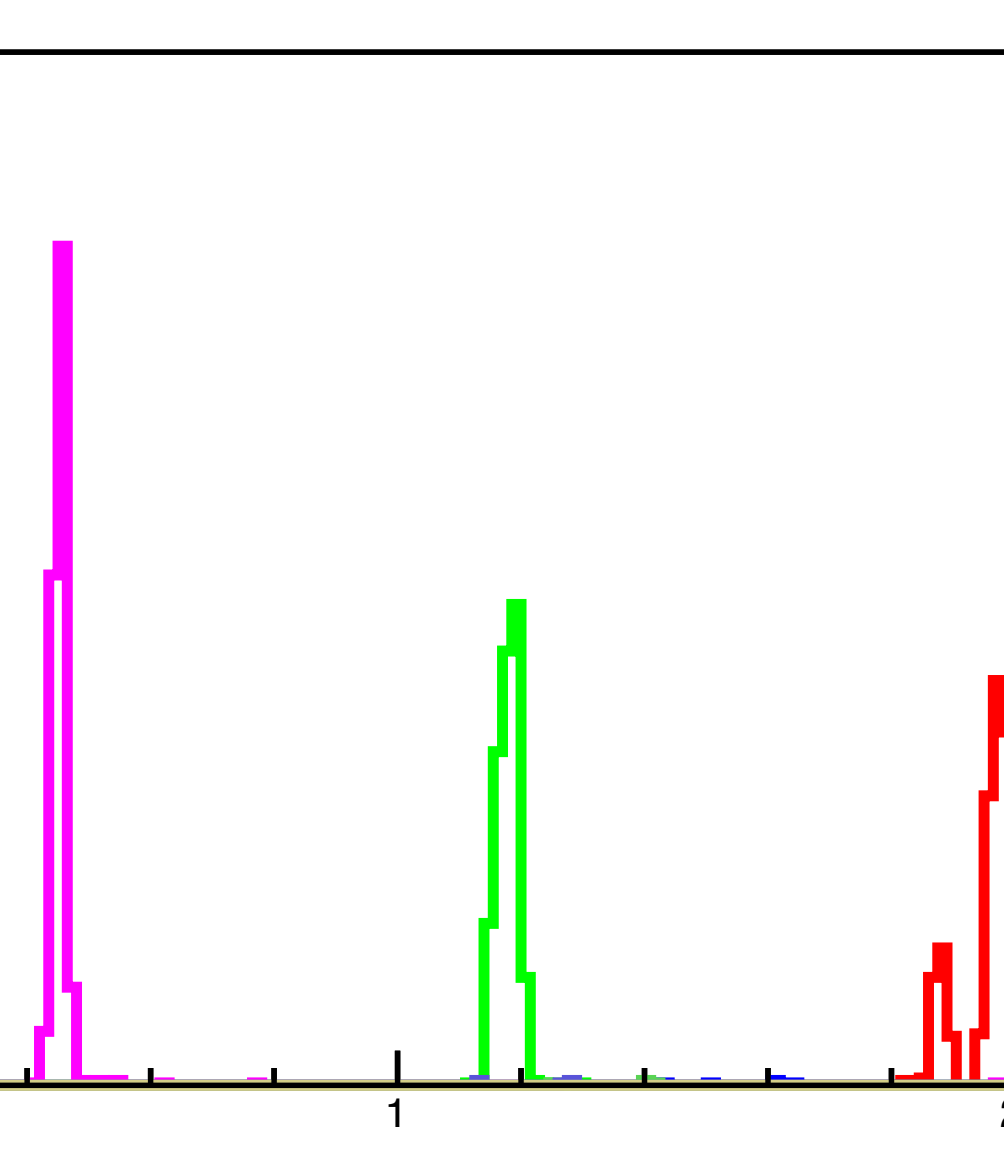

VELO Upgrade

Prototype Sensors

Timepix3 Telescope

- Time Resolution

Pointing Resolution

Rate Performance

Sensor Characterisation

HV Tolerance

Charge Collection

Efficiency

Spatial Resolution

Edge

- Grazing Angles

- Summary

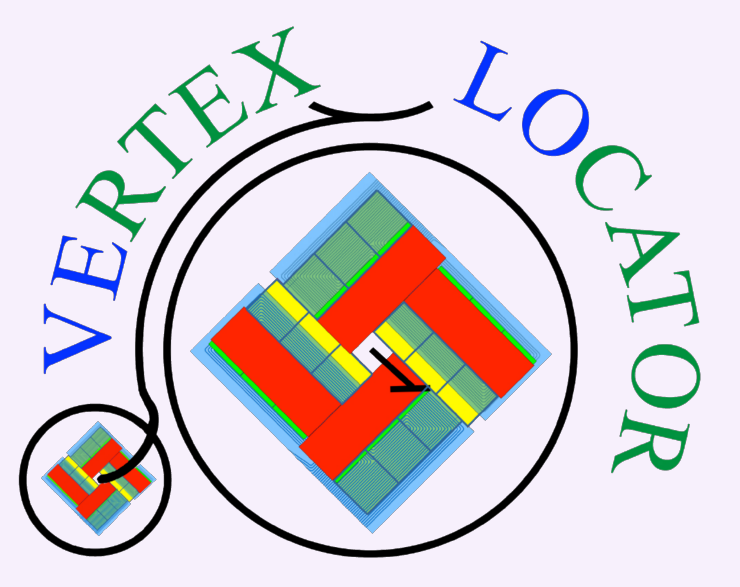


- fraction of non-associated clusters studied as a function of rate

- no significant dependence found

- rate limited by the SPS beam: the telescope should be able to operate at least twice the maximum rate

No degradation in efficiency up to the high rate of 5 million tracks / s / $\mathrm{cm}^{2}$

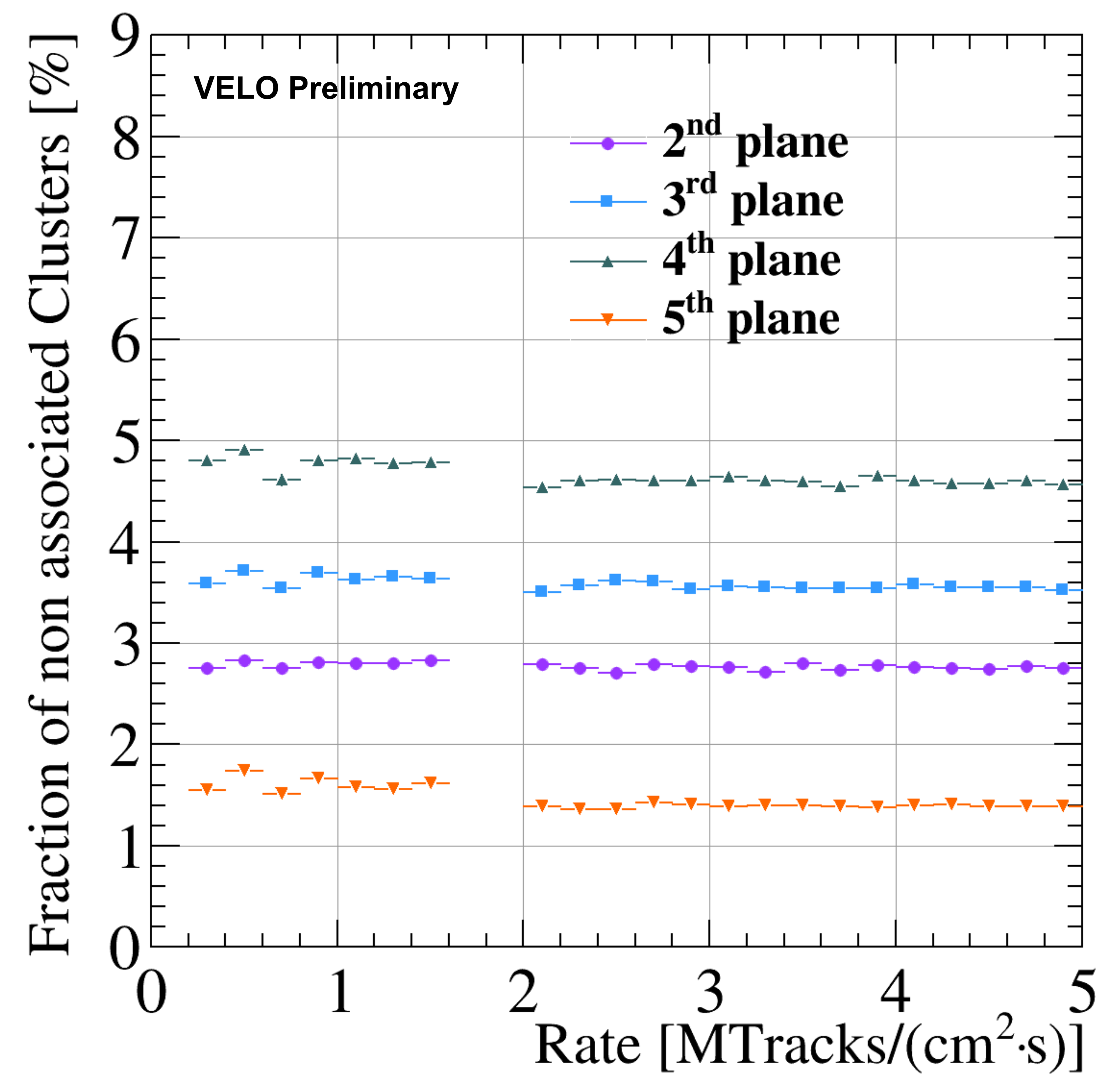

VELO Upgrade

Prototype Sensors

Timepix3 Telescope

- Time Resolution

Pointing Resolution

Rate Performance

Sensor Characterisation

HV Tolerance

Charge Collection

Efficiency

Spatial Resolution

Edge

Grazing Angles

Summary

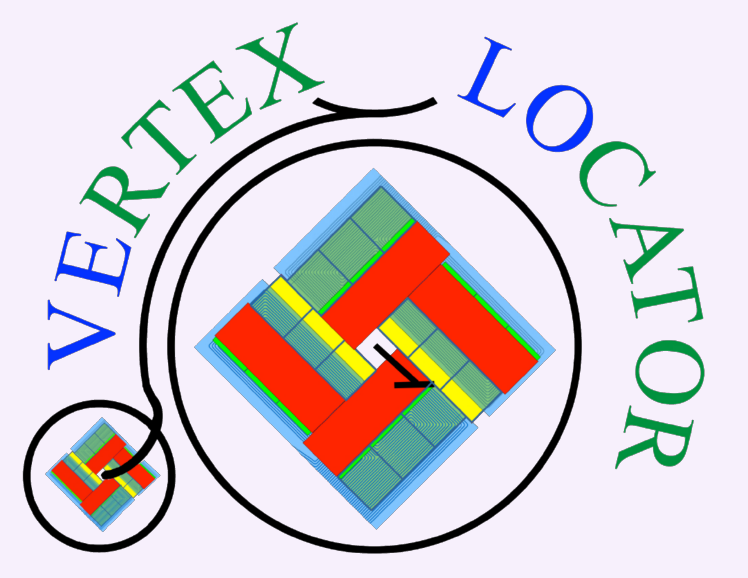


- VELO Upgrade

Prototype Sensors

Timepix3 Telescope

- Time Resolution

Pointing Resolution

Rate Performance

Sensitivity at the Edge

Spatial

Charge Collection Efficiency
Resolution

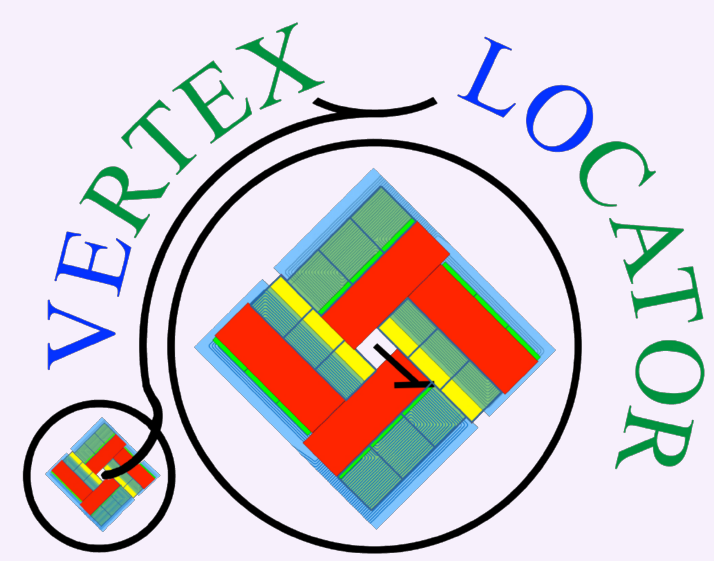




\section{sensors must withstand $1000 \mathrm{~V}$ after full fluence} (in both hottest and coolest part)

typical fluence profile at IRRAD

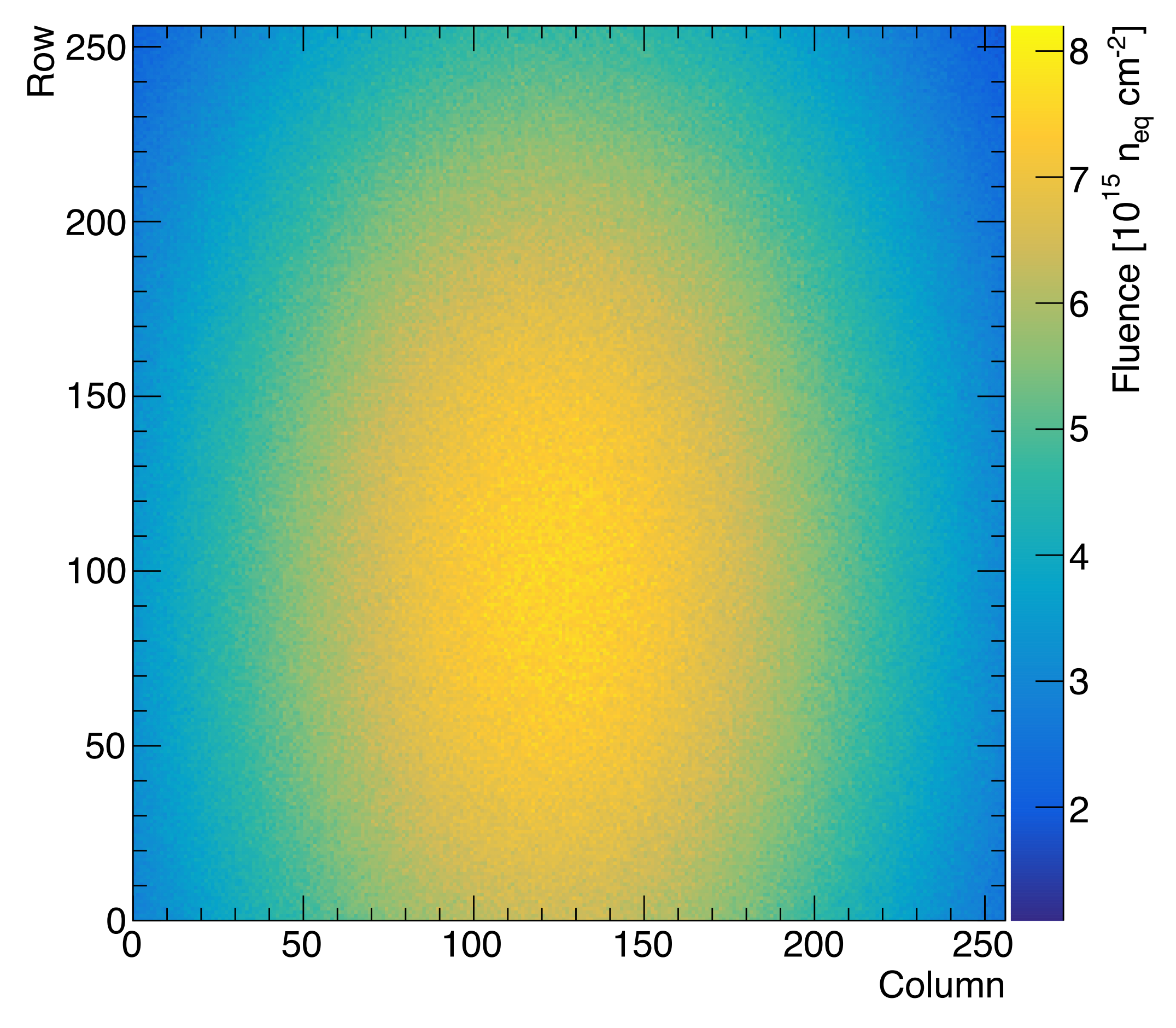

sensors non-uniformly proton irradiated at IRRAD

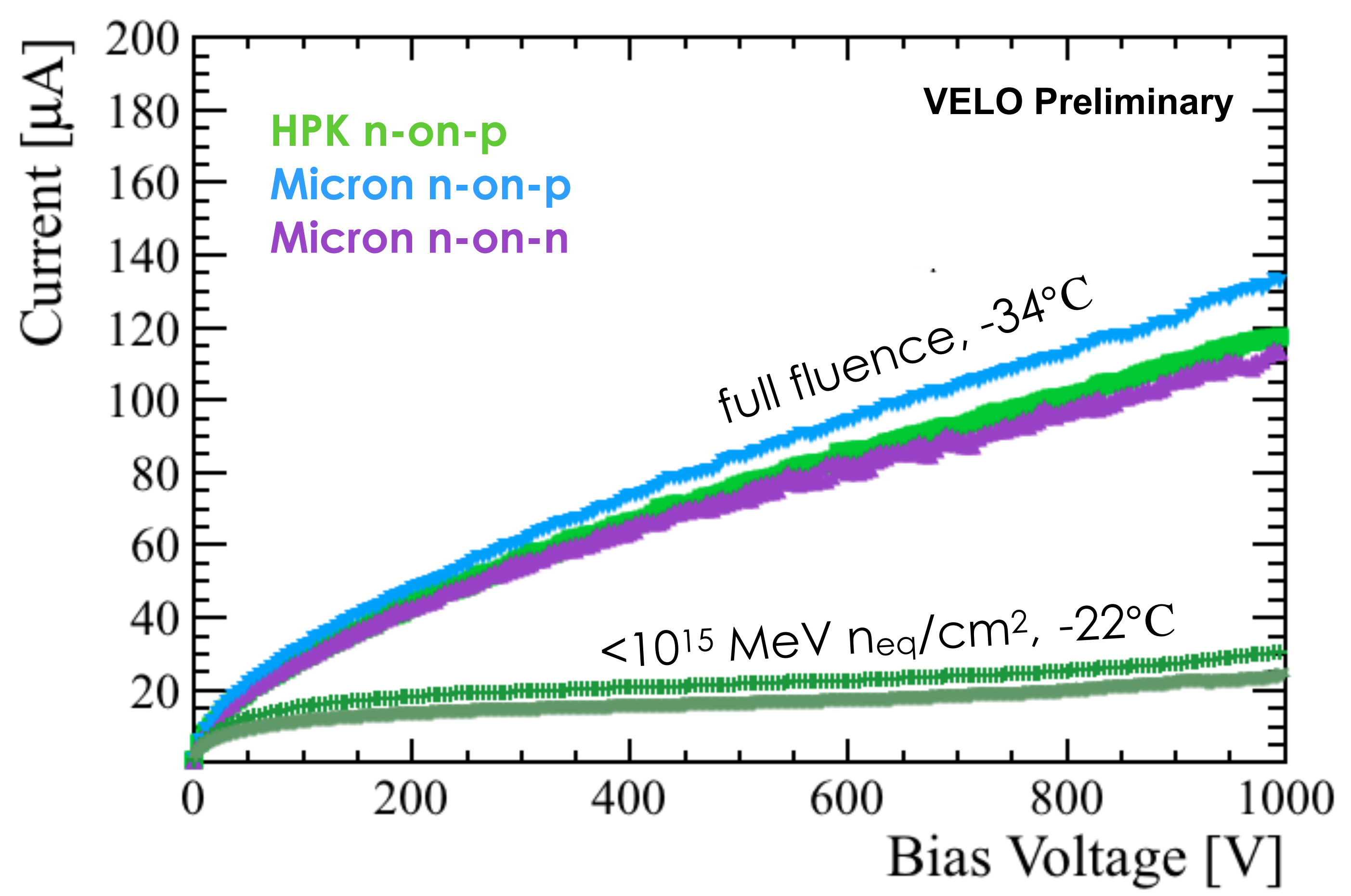

VELO Upgrade

Prototype Sensors

Timepix3 Telescope

- Time Resolution

Pointing Resolution

Rate Performance

- Sensor Characterisation

- HV Tolerance

- Charge Collection

- Efficiency

- Spatial Resolution

- Edge

- Grazing Angles

- Summary

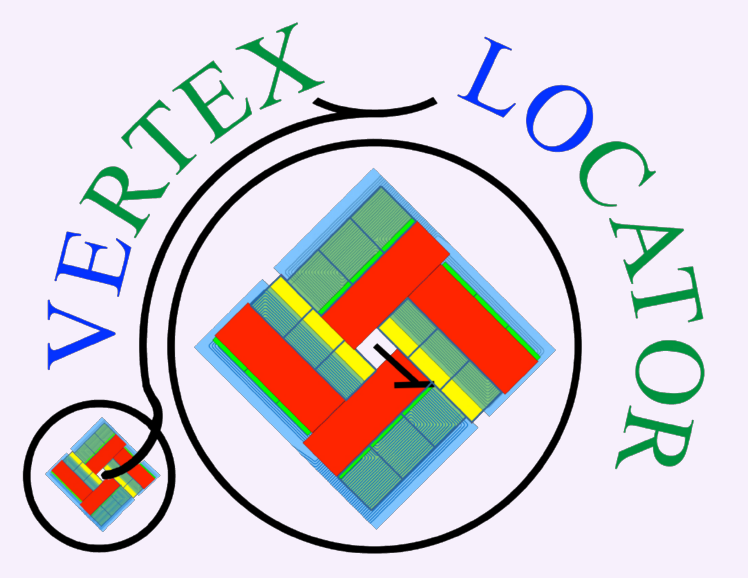


sensors uniformly neutron irradiated at JSI at full fluence

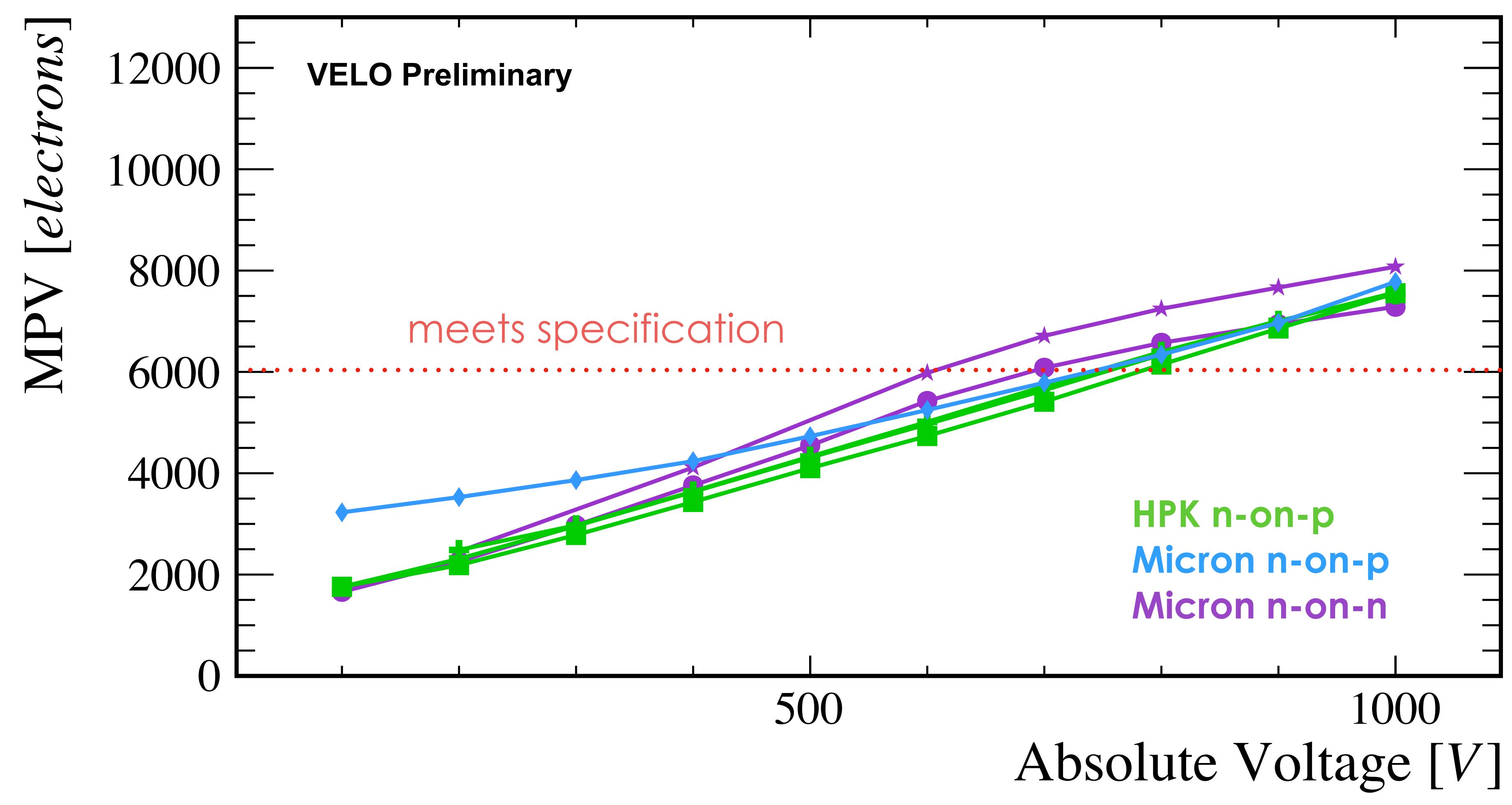

- Rate Performance

- Sensor Characterisation - HV Tolerance

- Charge Collection

- Efficiency

- Spatial Resolution

- Edge

- Grazing Angles

Summary

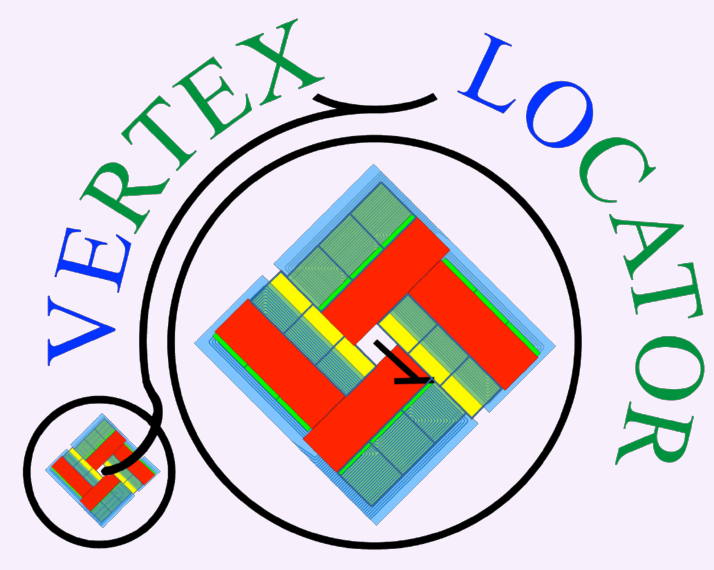




\section{efficiency must be $>99 \%$ everywhere in the sensor}

- $\mathrm{e}=$ nDUT_Associated_Clusters / nTracks

- non irradiated assemblies are fully efficient over the entire pixel cell

- irradiated assemblies show inefficiencies at pixel corners at lower applied $\vee$, but are fully efficient at $1000 \mathrm{~V}$

Pixel matrix

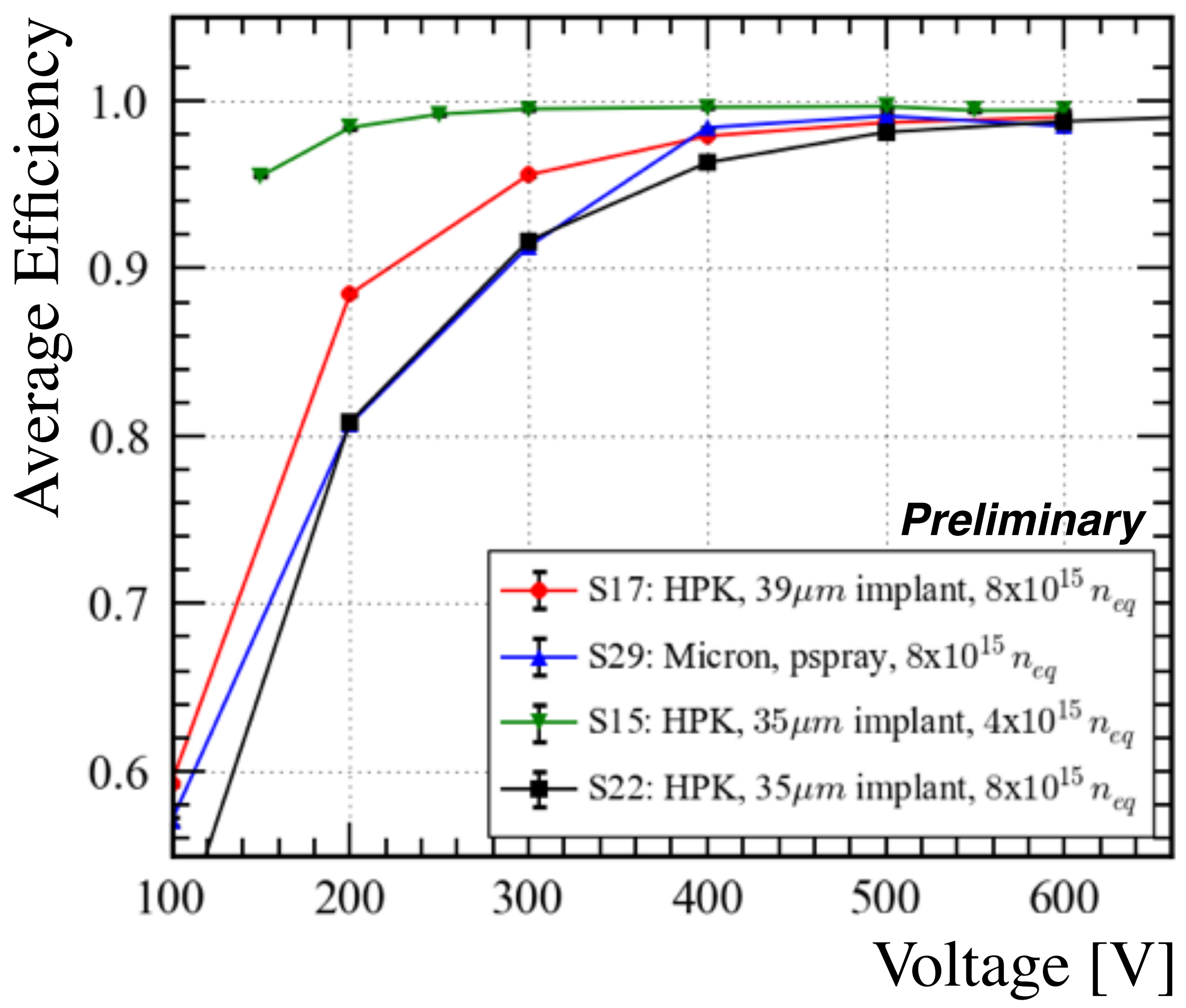

VELO Upgrade

Prototype Sensors

- Timepix3 Telescope

Time Resolution

Pointing Resolution

Rate Performance

- Sensor Characterisation HV Tolerance

- Charge Collection

- Efficiency

- Spatial Resolution

- Edge

- Grazing Angles

- Summary

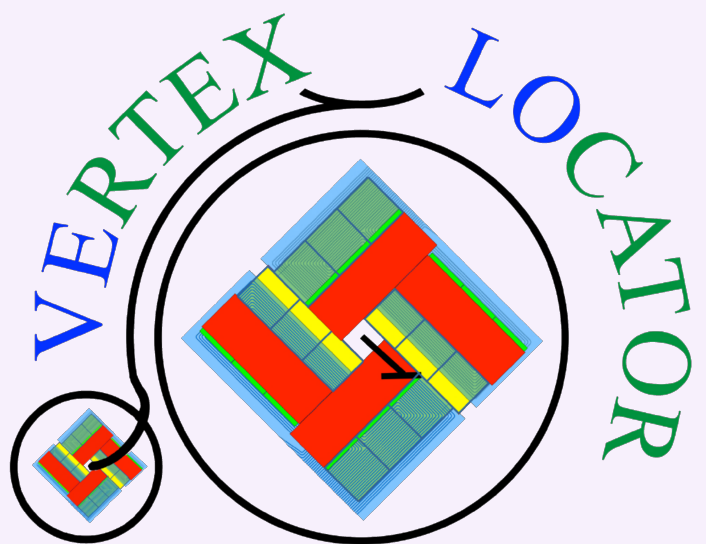



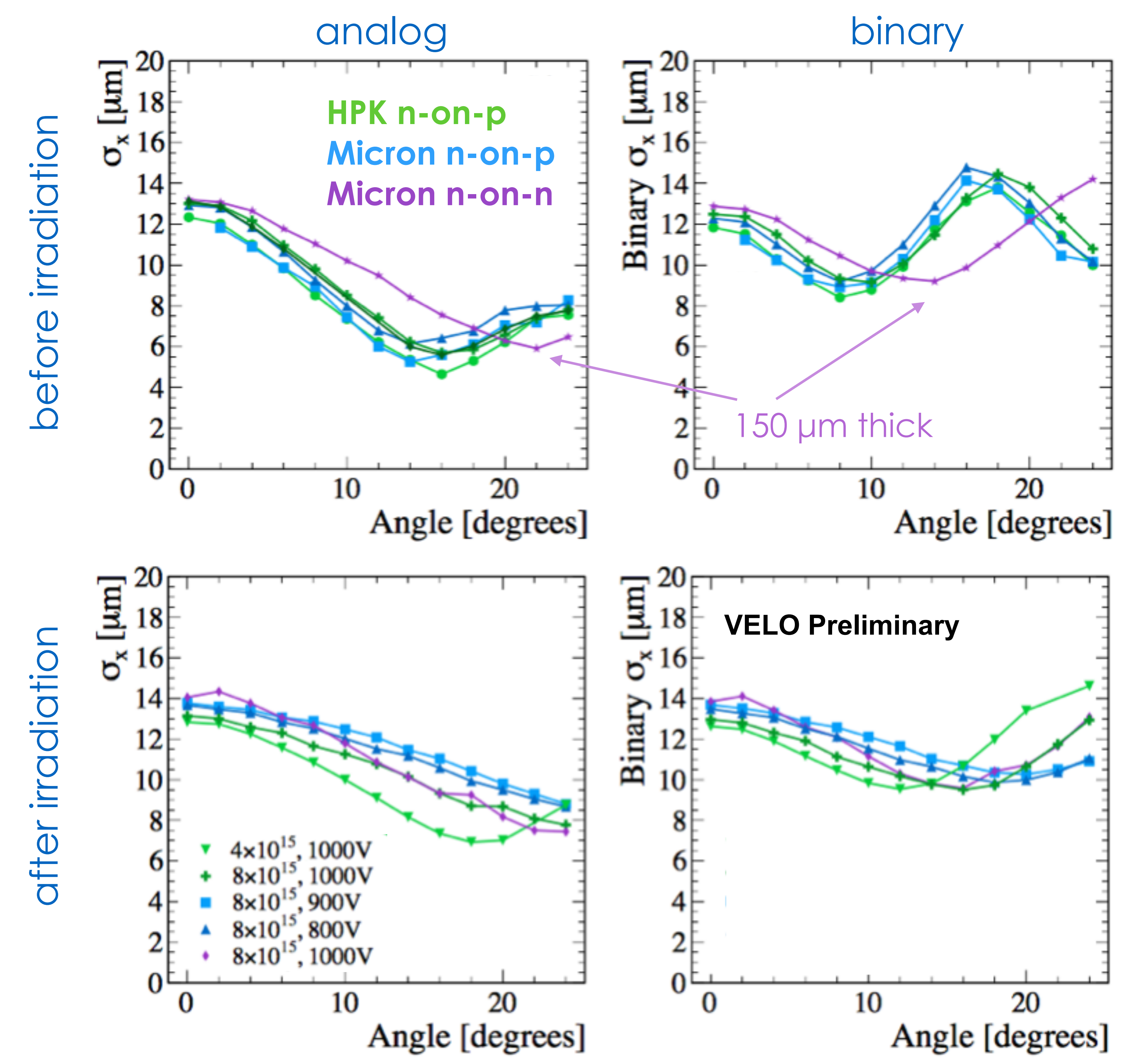

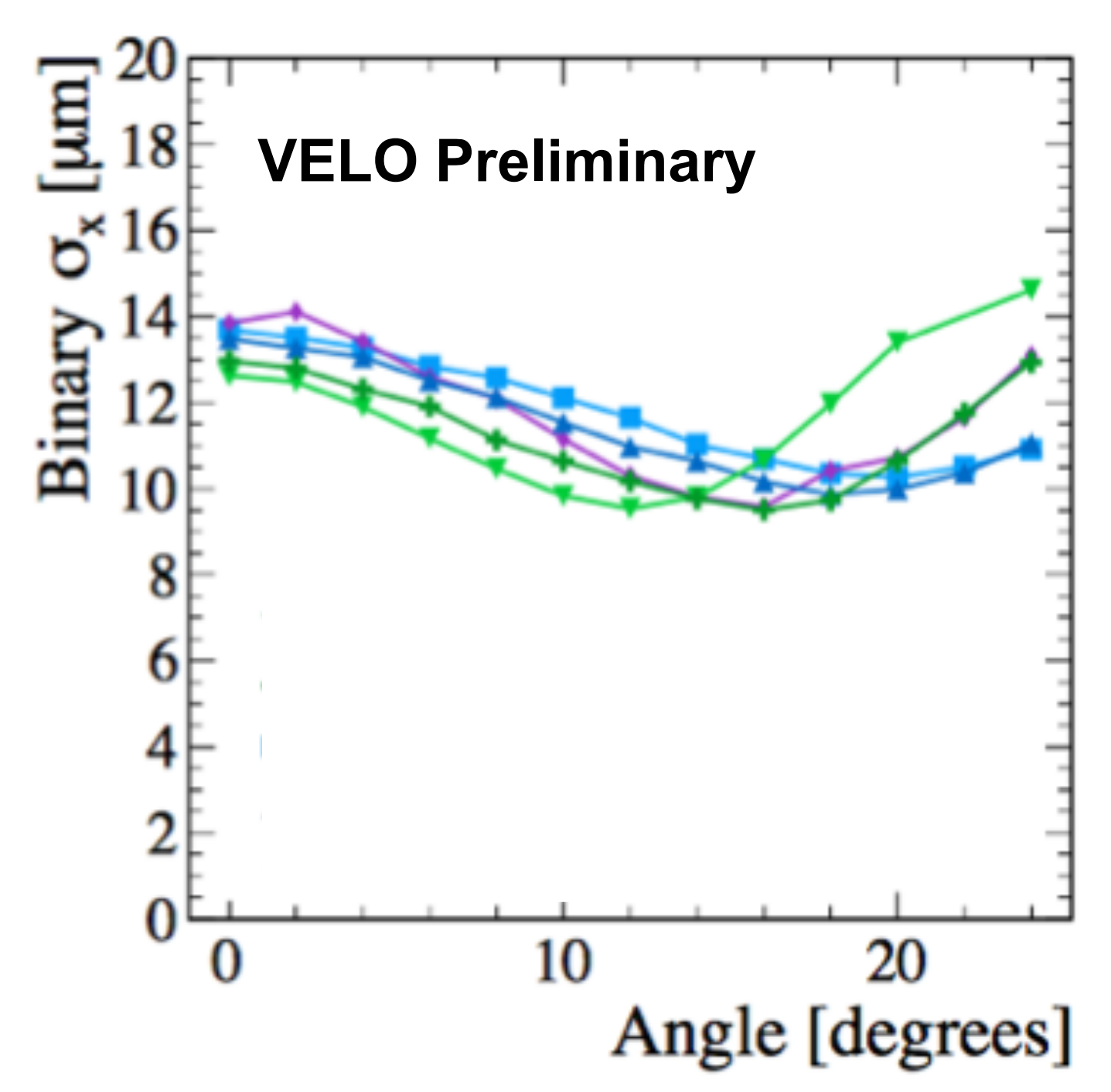

\section{spatial resolution must be excellent after full dose}

- analog resolution: position reconstructed using the Centre of Gravity technique

- binary resolution: position reconstructed ignoring the charge information
VELO Upgrade

Prototype Sensors

Timepix3 Telescope

Time Resolution

Pointing Resolution

Rate Performance

- Sensor Characterisation

HV Tolerance

Charge Collection

Efficiency

spatial Resolution

- Edge

- Grazing Angles

- Summary

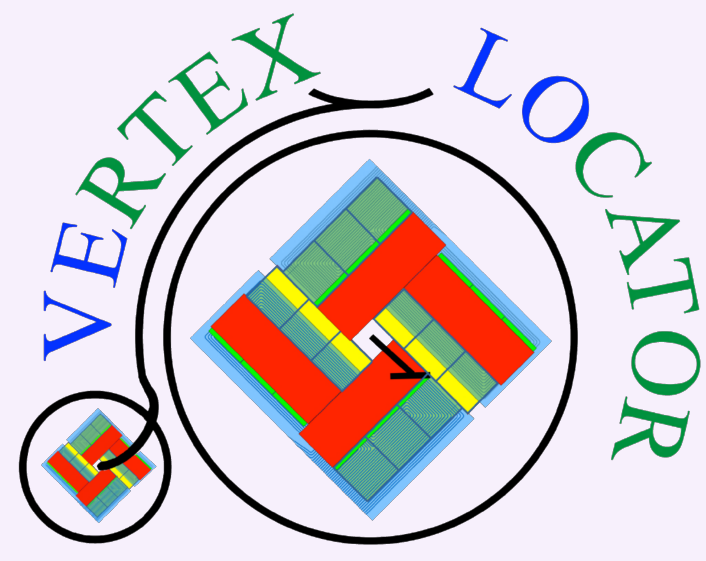




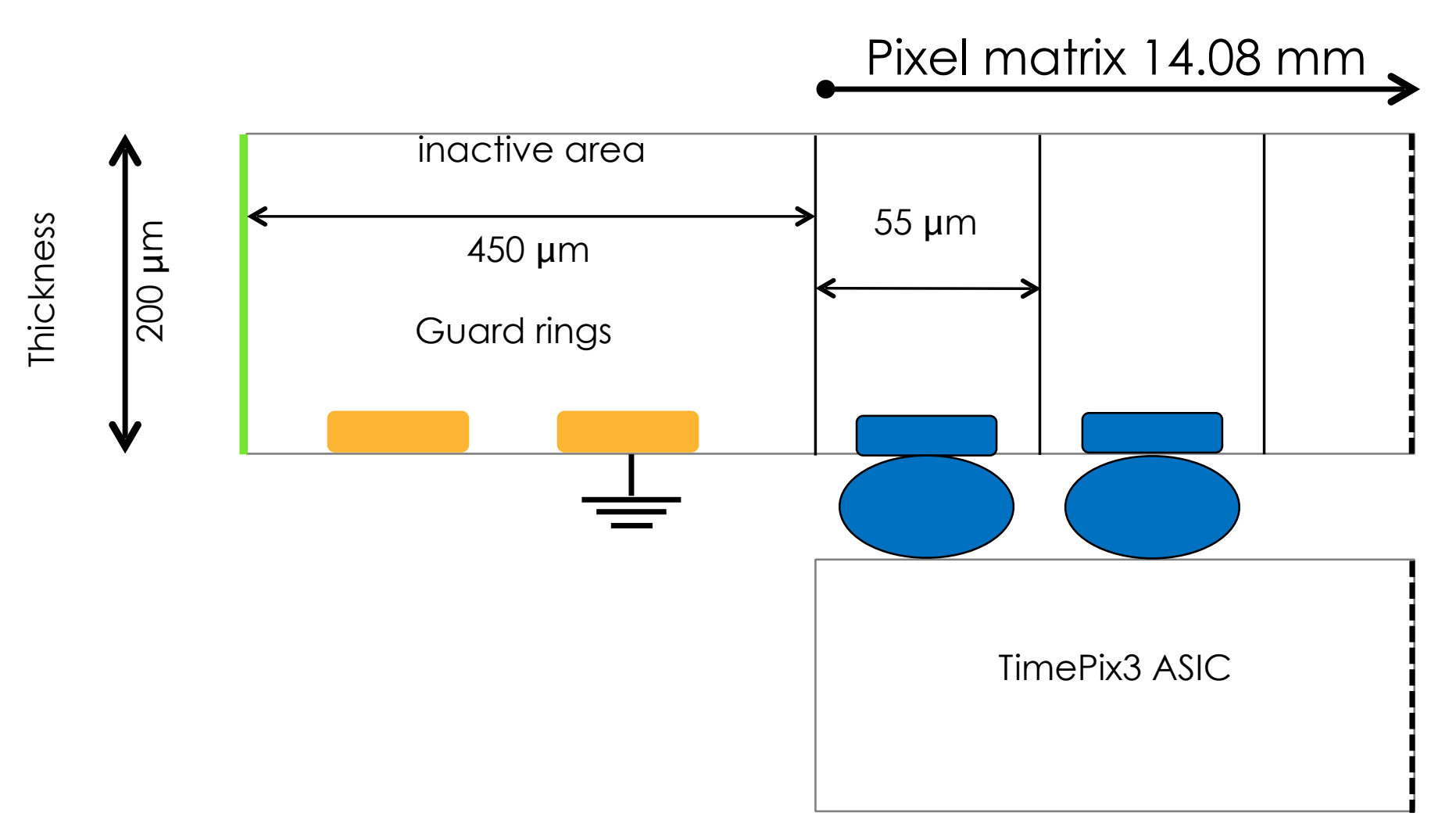

sensor must be fully efficient up to edge

Micron n-on-p

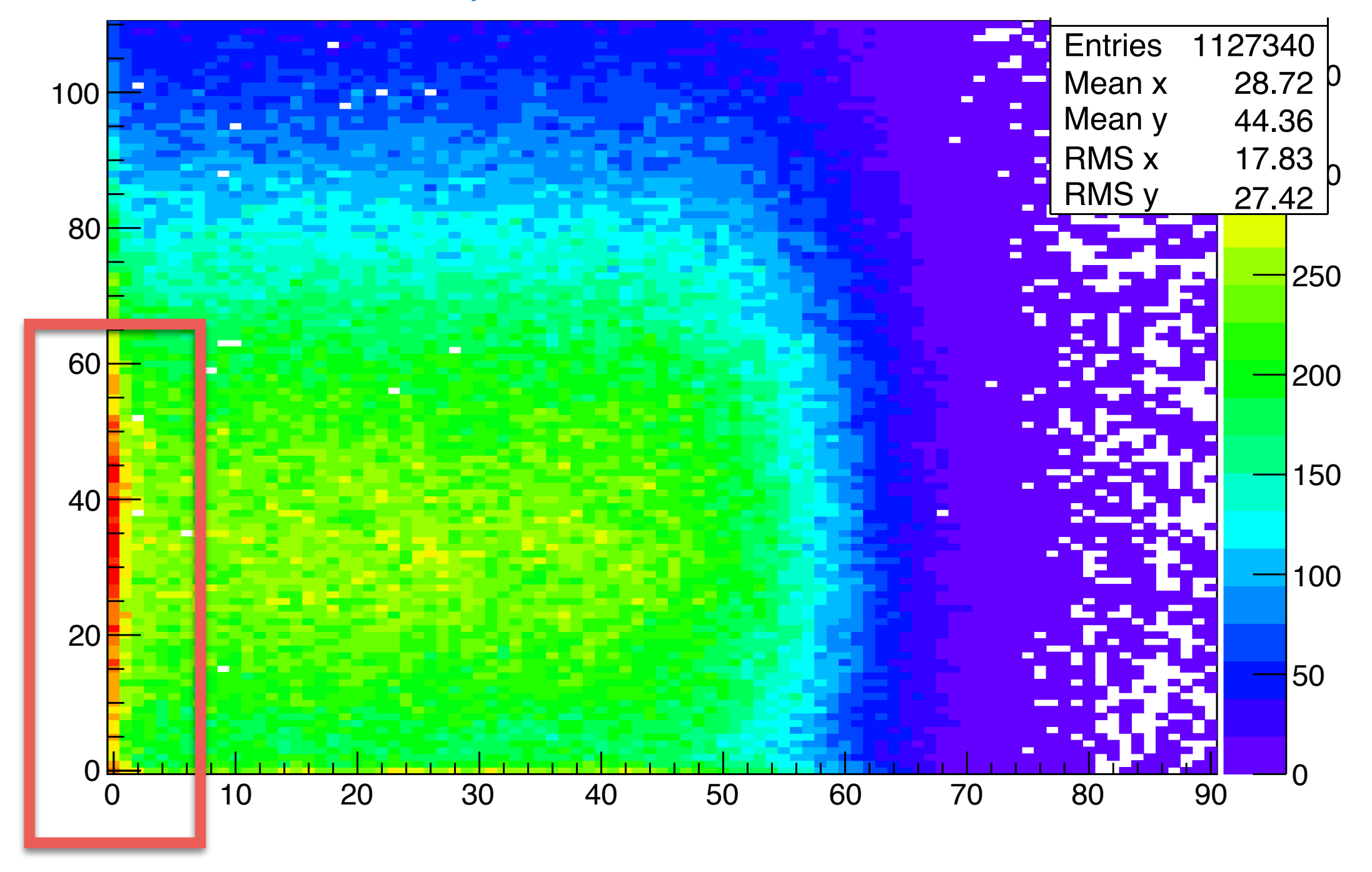

- Guard ring structure to gradually reduce the electric field towards the edge of the sensor

- Occupancy is already higher close to edge

- Distortion of the electric field that would lead to a worst spatial resolution must be avoided

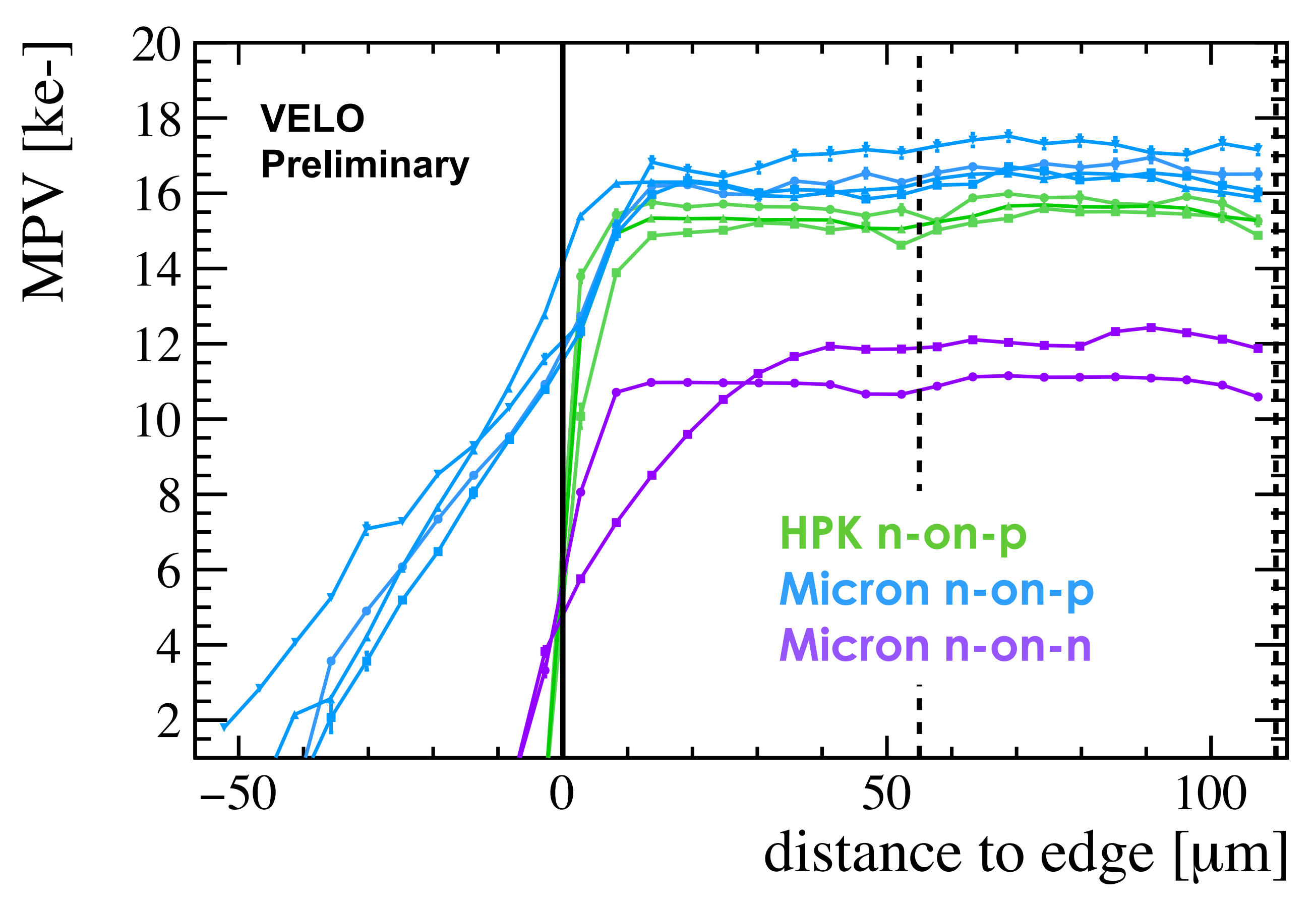

VELO Upgrade

Prototype Sensors

Timepix3 Telescope

- Time Resolution

- Pointing Resolution

- Rate Performance

- Sensor Characterisation

- HV Tolerance

- Charge Collection

- Efficiency

- Spatial Resolution

- Edge

- Grazing Angles

- Summary

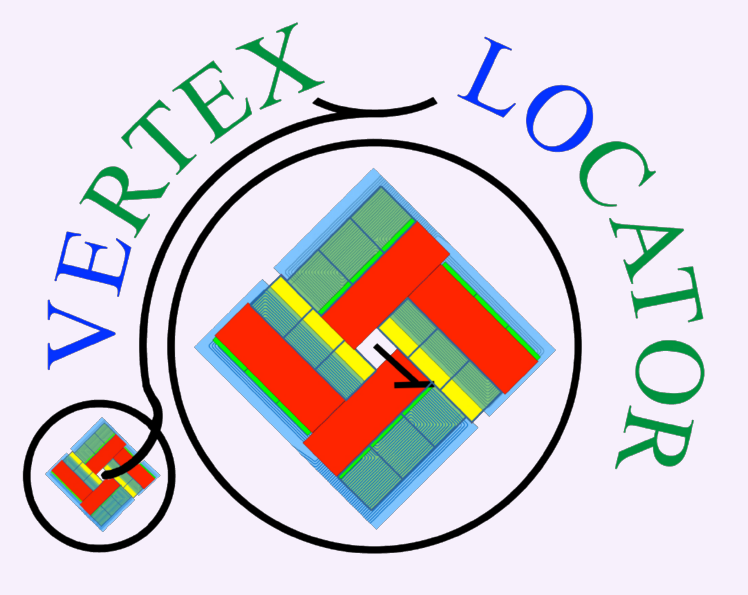


The sensor is rotated at a very large angle (83-89 deg) with respect to the beam

Exploiting the relation between the hit pixel position in the cluster and the depth sampled by the track:

- Charge collection as a function of depth

- Time required to cross the threshold as a function of depth

\section{Non-irradiated}

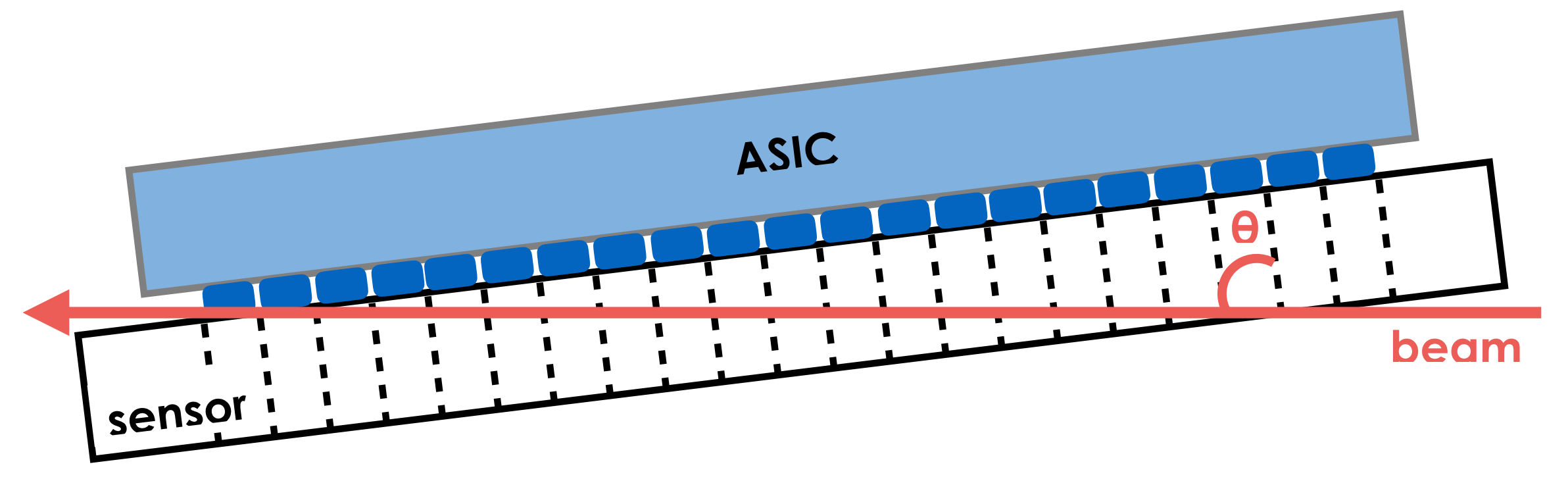

VELO Upgrade

Prototype Sensors

Timepix3 Telescope

Time Resolution

Pointing Resolution

Rate Performance

- Sensor Characterisation

- HV Tolerance

- Charge Collection

- Efficiency

Spatial Resolution

Edge

- Grazing Angles

Summary

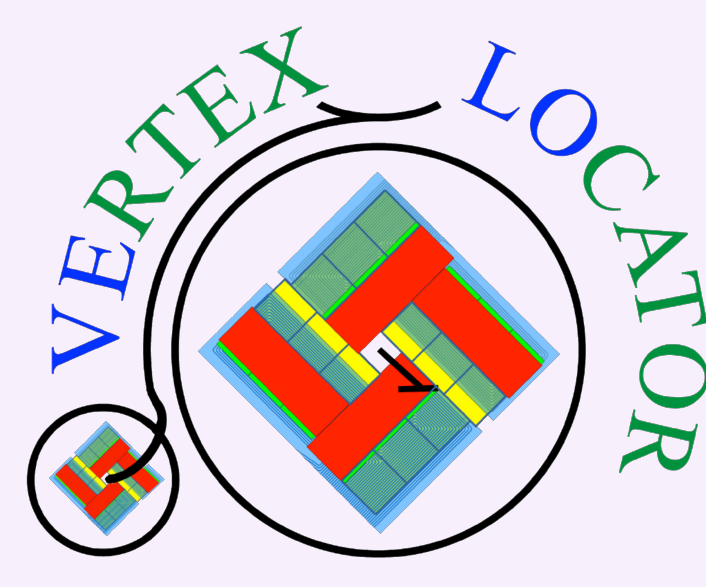


The sensor is rotated at a very large angle (83-89 deg) with respect to the beam

Exploiting the relation between the hit pixel position in the cluster and the depth sampled by the track:

- Charge collection as a function of depth

- Time required to cross the threshold as a function of depth

Uniformly neutron irradiated ( 8 x $\left.10151 \mathrm{MeV}_{\mathrm{eq}} \mathrm{cm}^{-2}\right)$

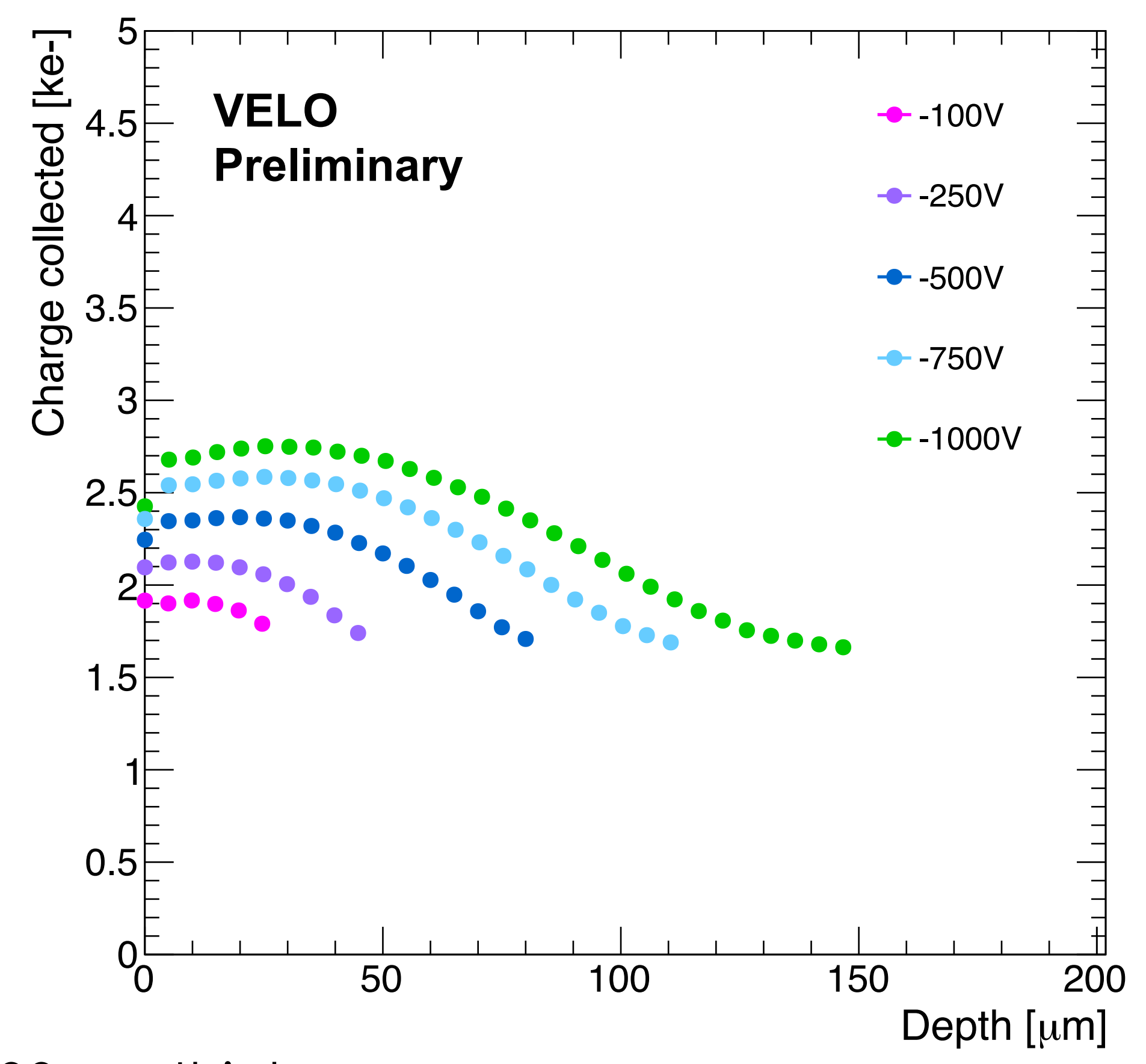

HPK n-on-p, $200 \mu m$ thick

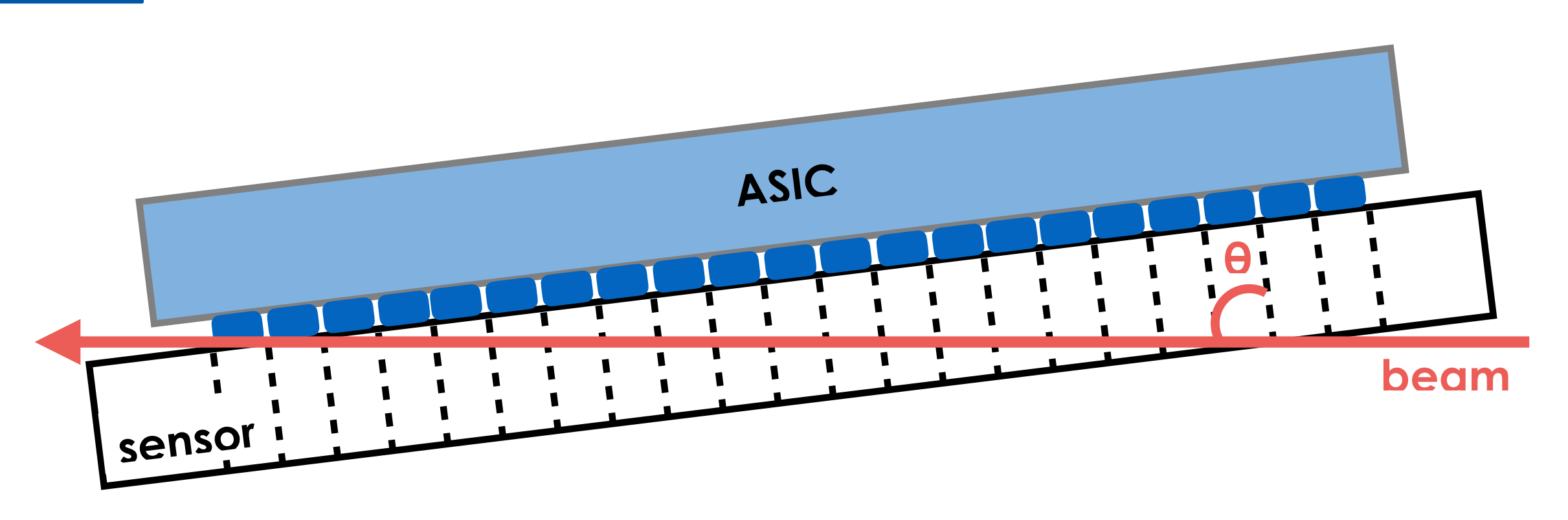

VELO Upgrade

Prototype Sensors

Timepix3 Telescope

Time Resolution

Pointing Resolution

Rate Performance

- Sensor Characterisation

- HV Tolerance

Charge Collection

Efficiency

Spatial Resolution

Edge

Grazing Angles

Summary

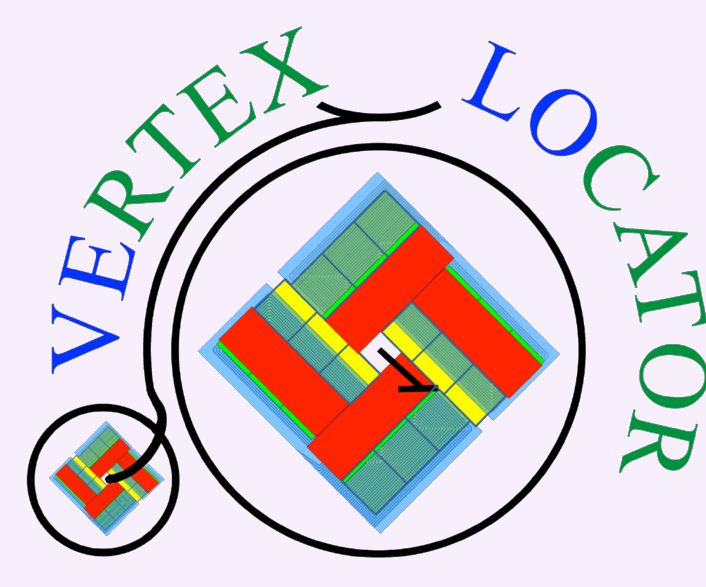




\section{Grazing Angles}

\section{Non uniformly proton irradiated full fluence}

typical fluence profile at IRRAD

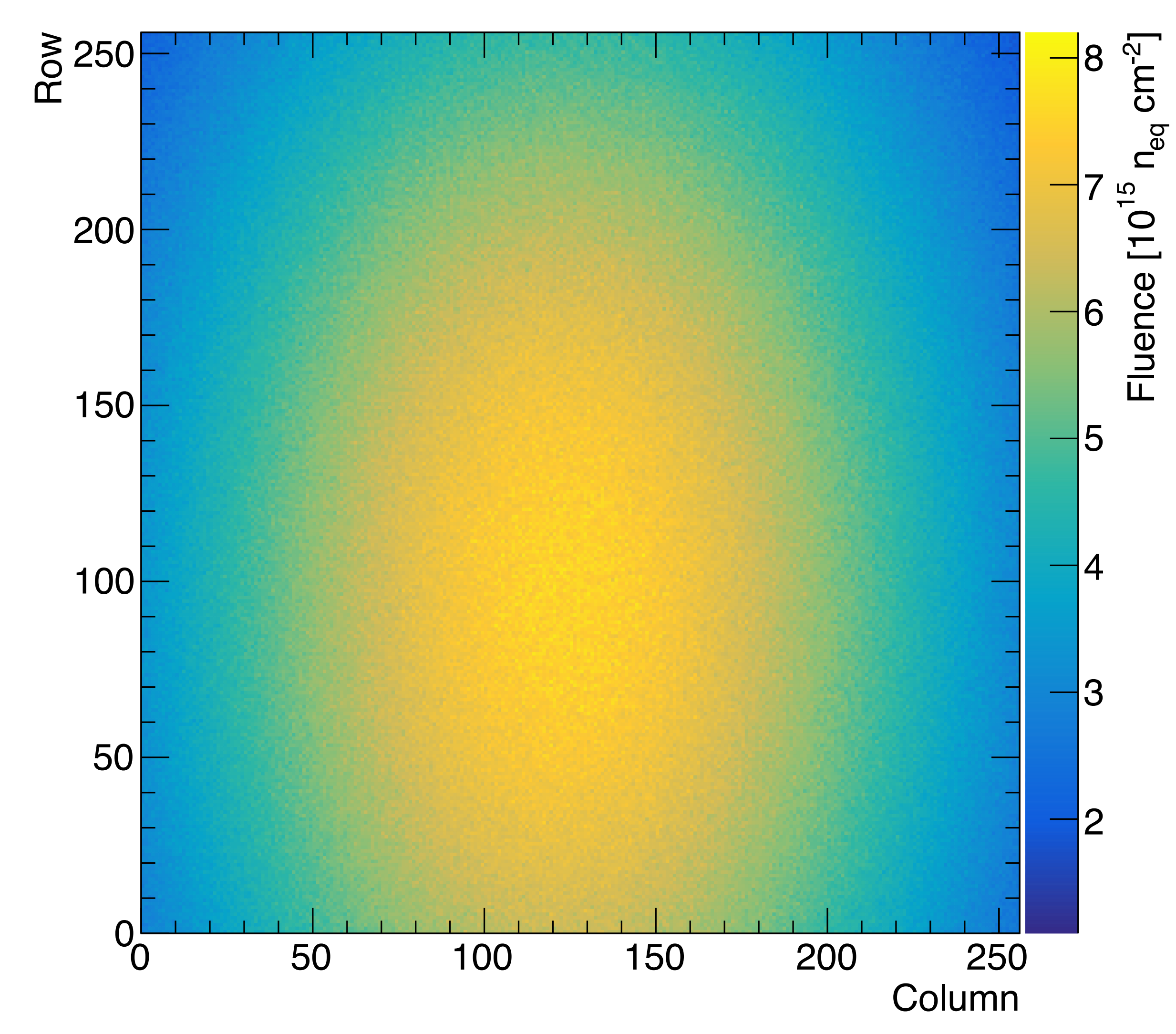

$250 \mathrm{~V}$

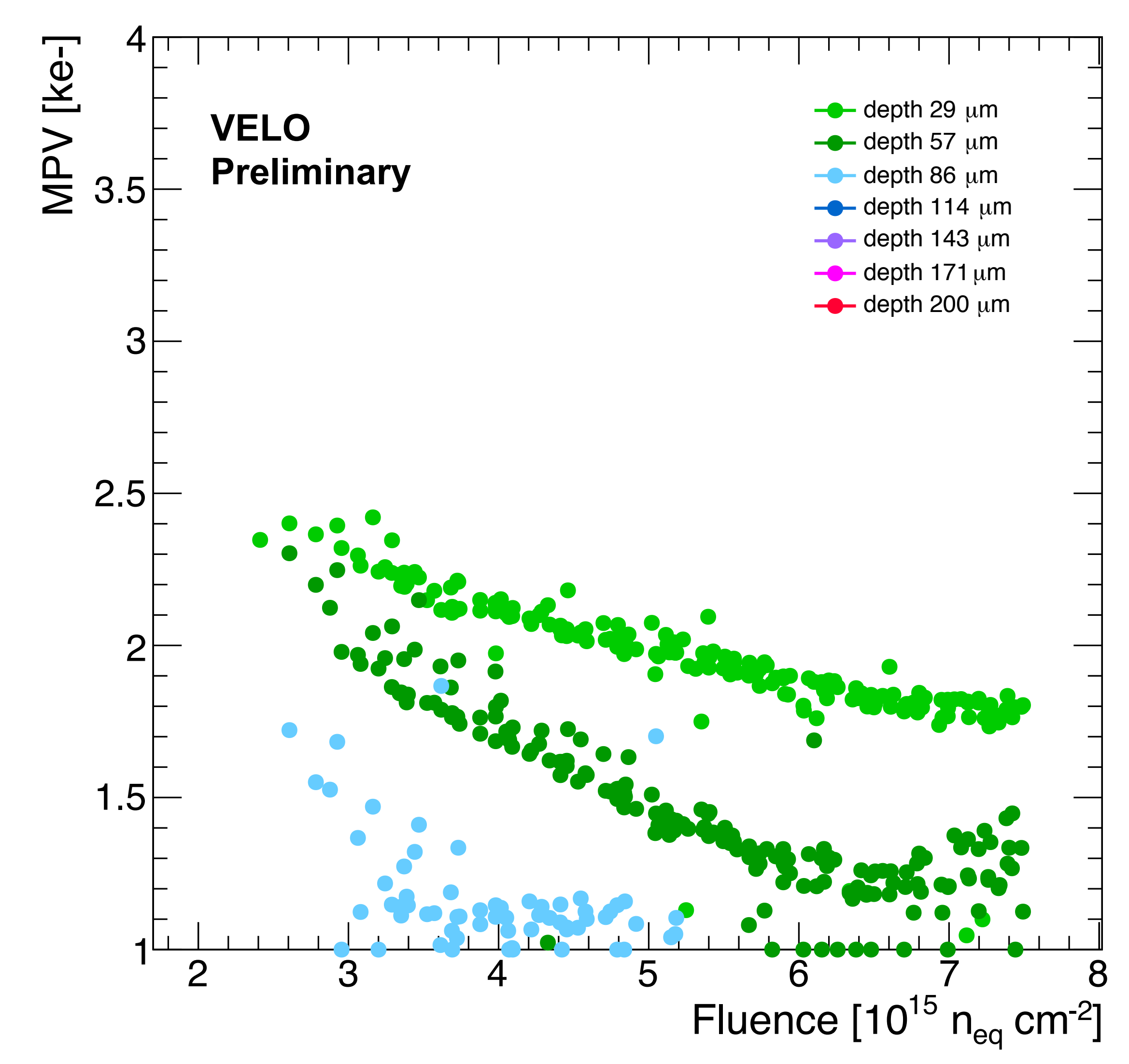

VELO Upgrade

Prototype Sensors

- Timepix3 Telescope

- Time Resolution

- Pointing Resolution

- Rate Performance

- Sensor Characterisation

- HV Tolerance

- Charge Collection

- Efficiency

- Spatial Resolution

- Edge

- Grazing Angles

- Summary

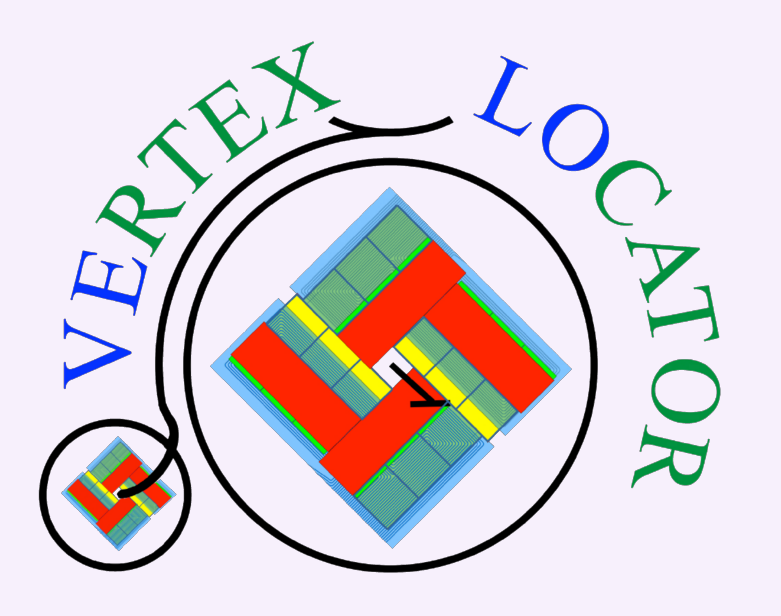




\section{Grazing Angles}

\section{Non uniformly proton irradiated full fluence}

typical fluence profile at IRRAD

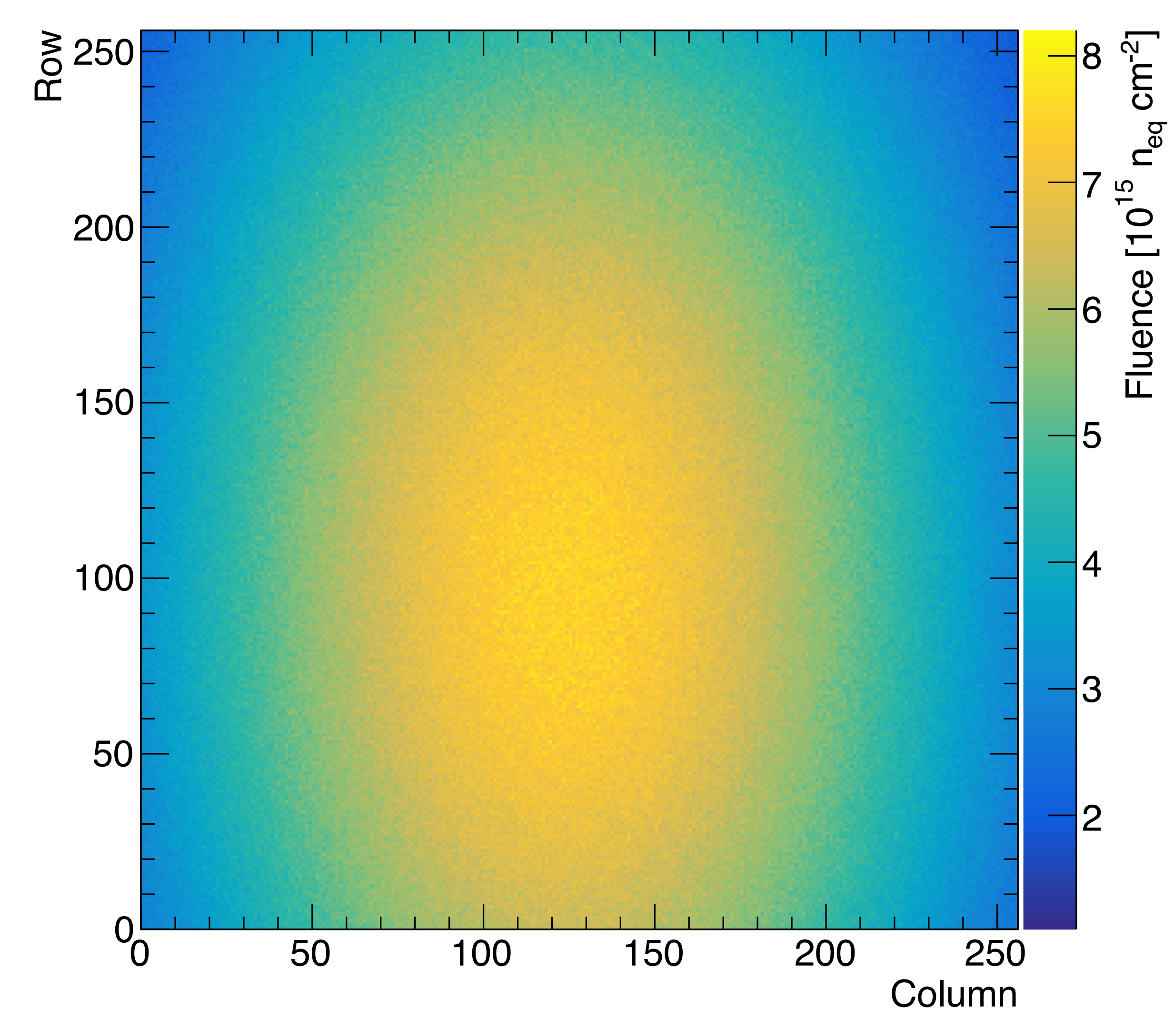

$500 \mathrm{~V}$

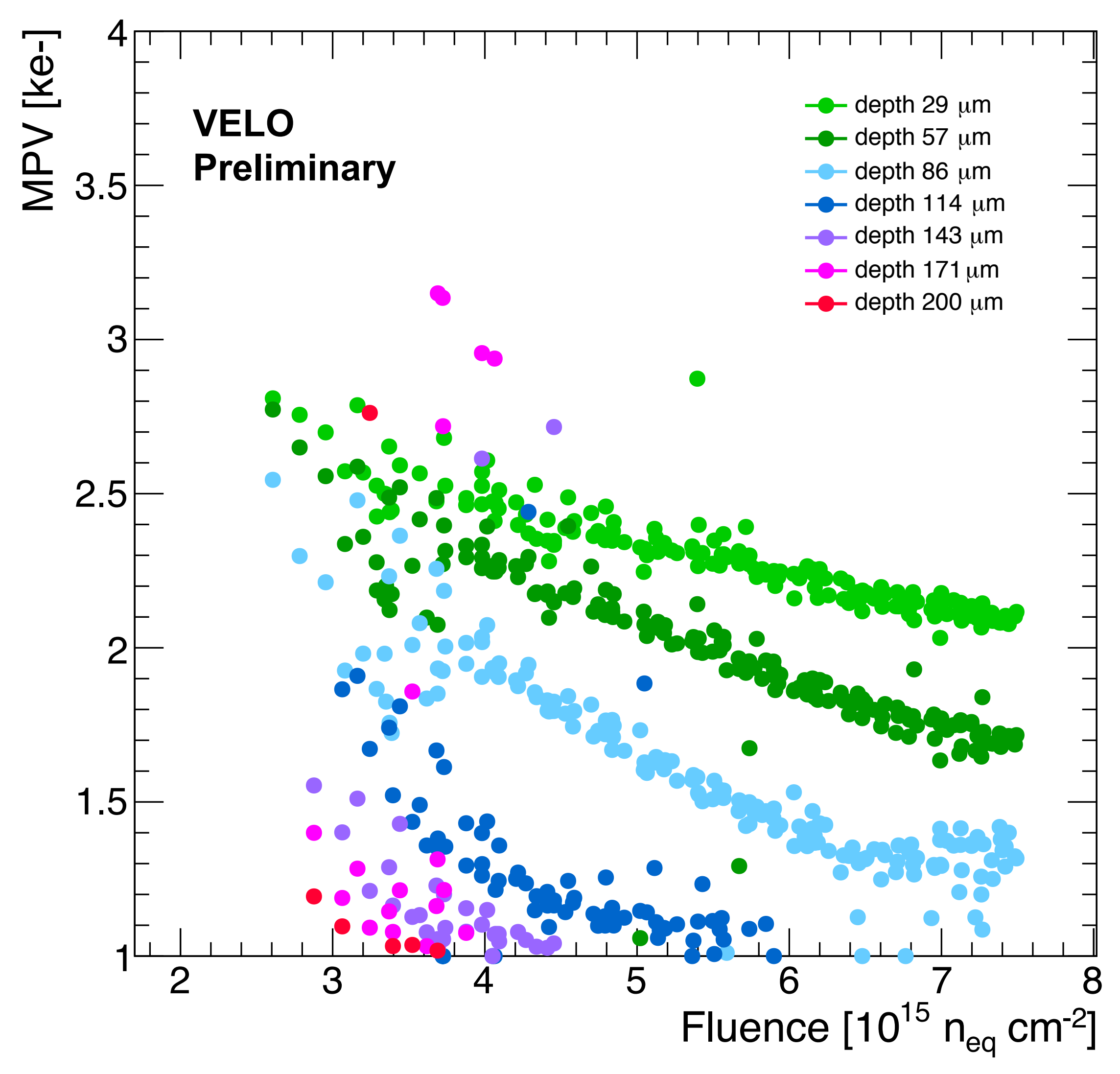

VELO Upgrade

Prototype Sensors

- Timepix3 Telescope

- Time Resolution

- Pointing Resolution

Rate Performance

- Sensor Characterisation

- HV Tolerance

Charge Collection

Efficiency

Spatial Resolution

Edge

Grazing Angles

- Summary

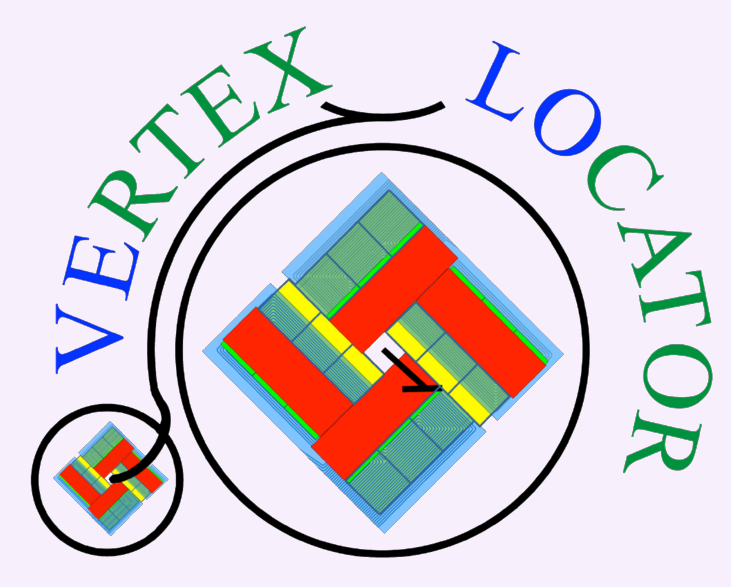




\section{Grazing Angles}

\section{Non uniformly proton irradiated full fluence}

typical fluence profile at IRRAD

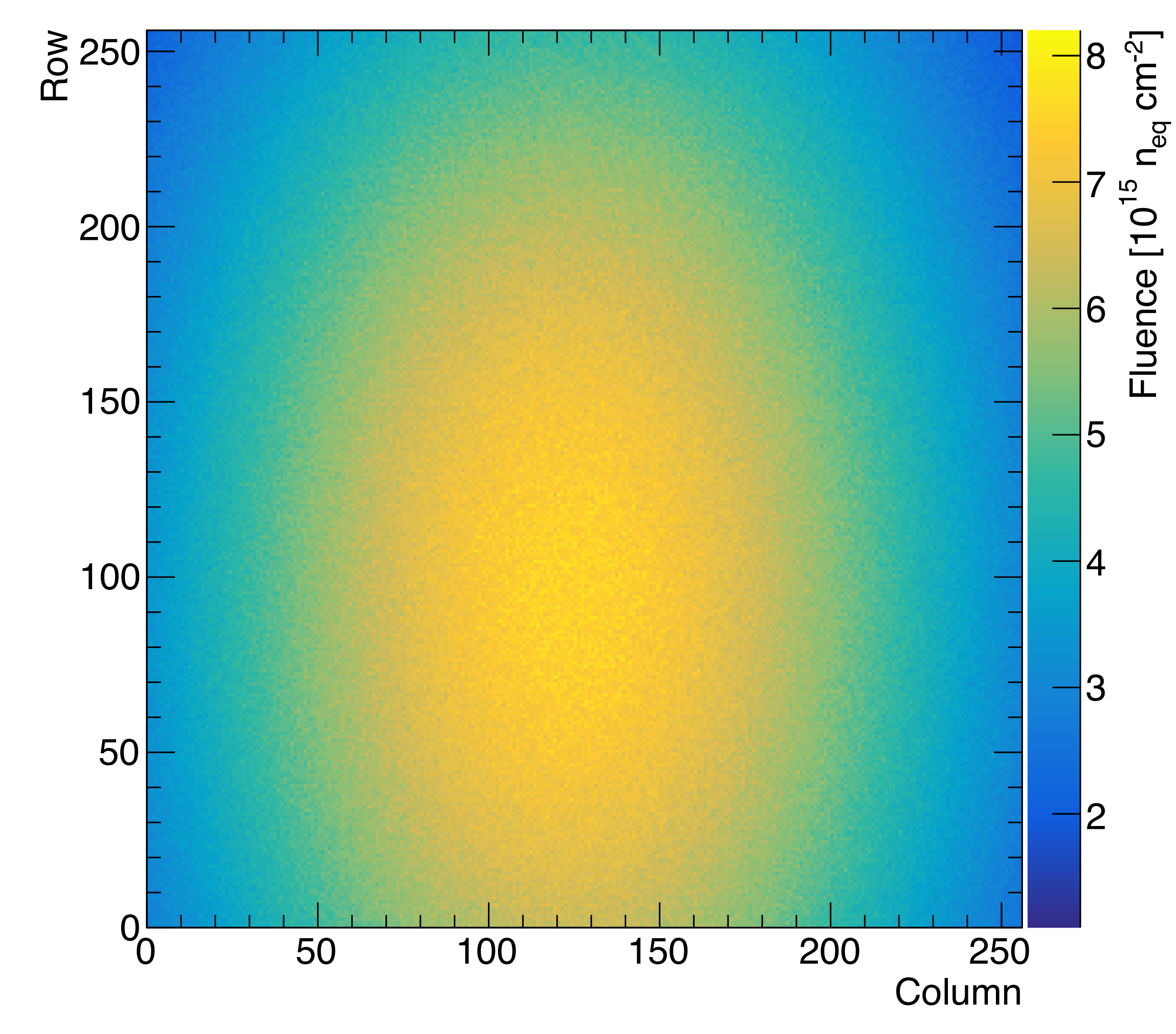

$750 \mathrm{~V}$

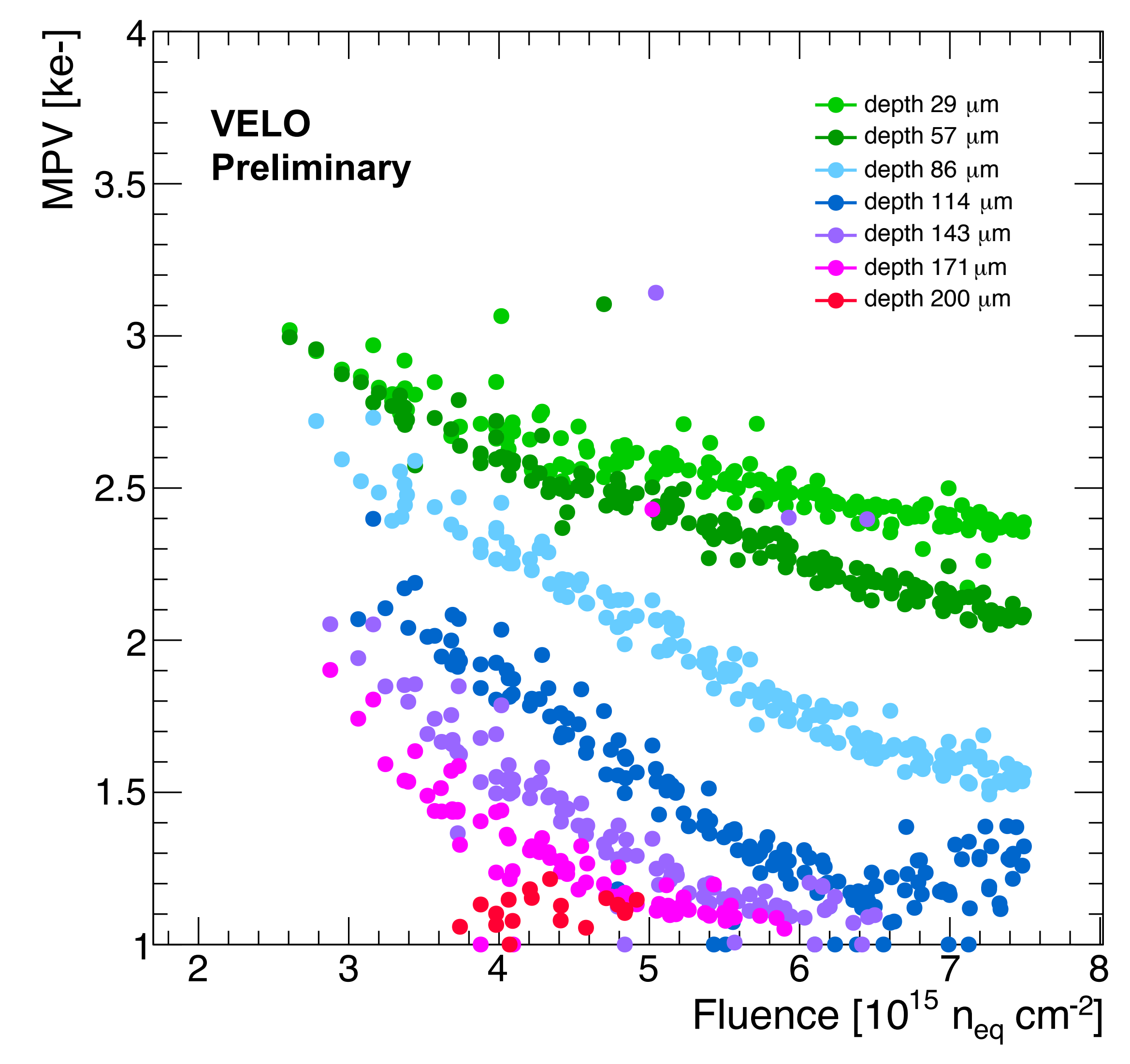

VELO Upgrade

Prototype Sensors

- Timepix3 Telescope

- Time Resolution

- Pointing Resolution

Rate Performance

- Sensor Characterisation

- HV Tolerance

- Charge Collection

- Efficiency

- Spatial Resolution

- Edge

- Grazing Angles

- summary

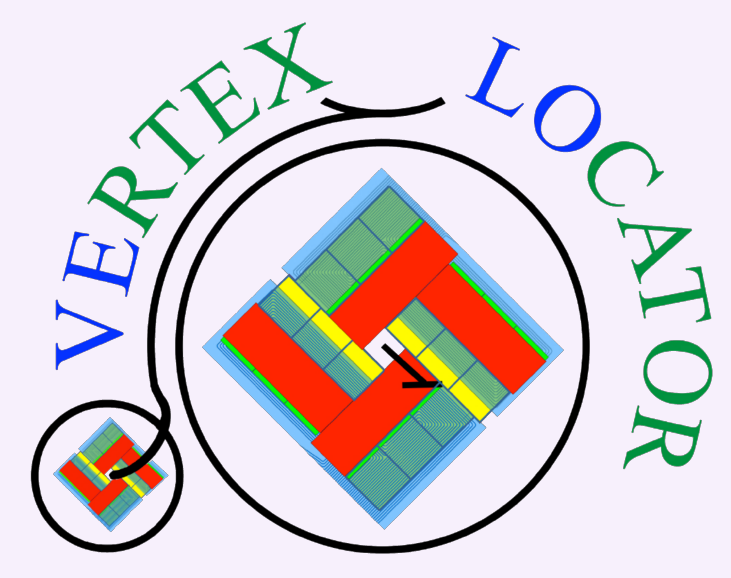




\section{Grazing Angles}

\section{Non uniformly proton irradiated full fluence}

typical fluence profile at IRRAD

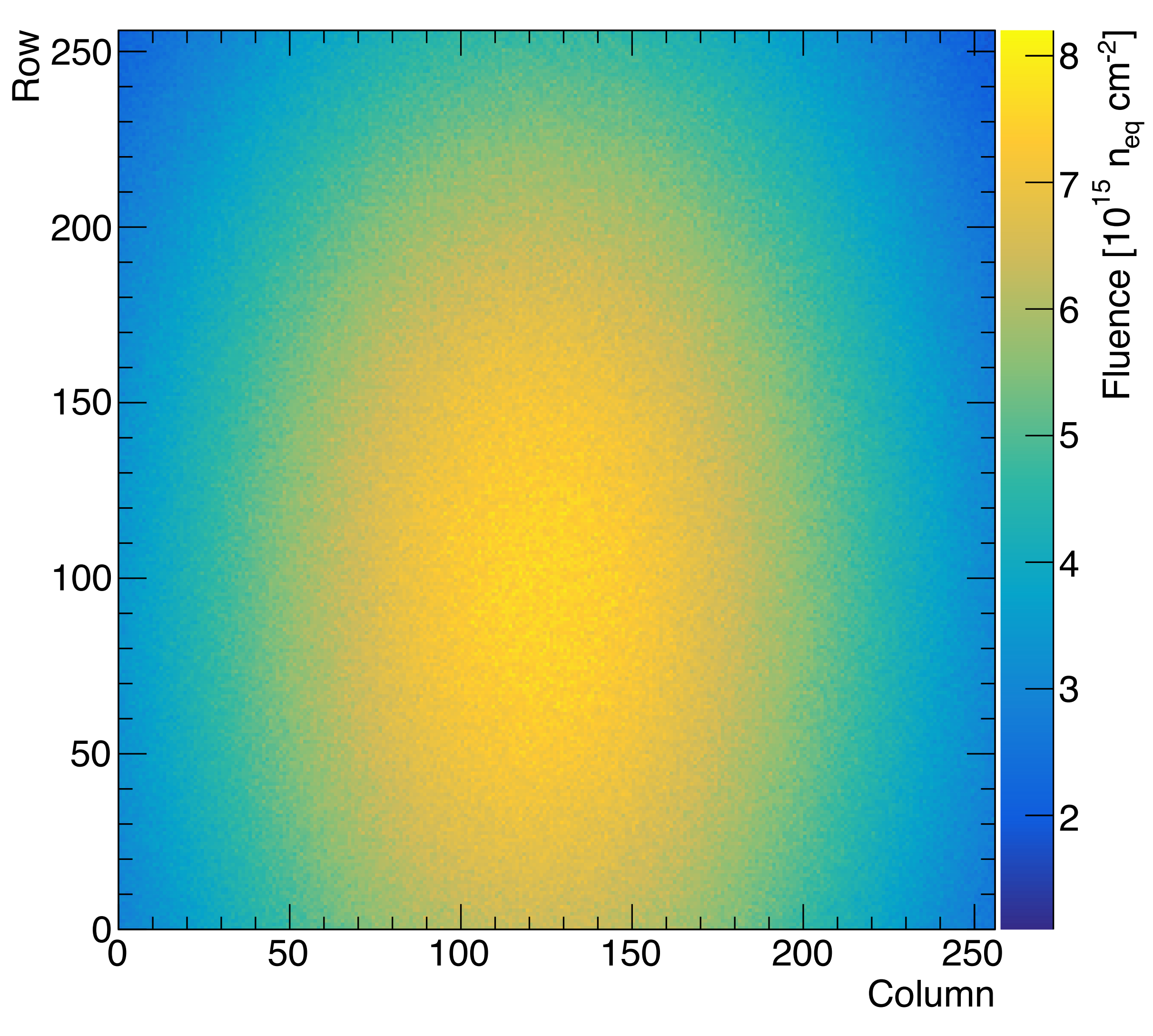

$1000 \mathrm{~V}$

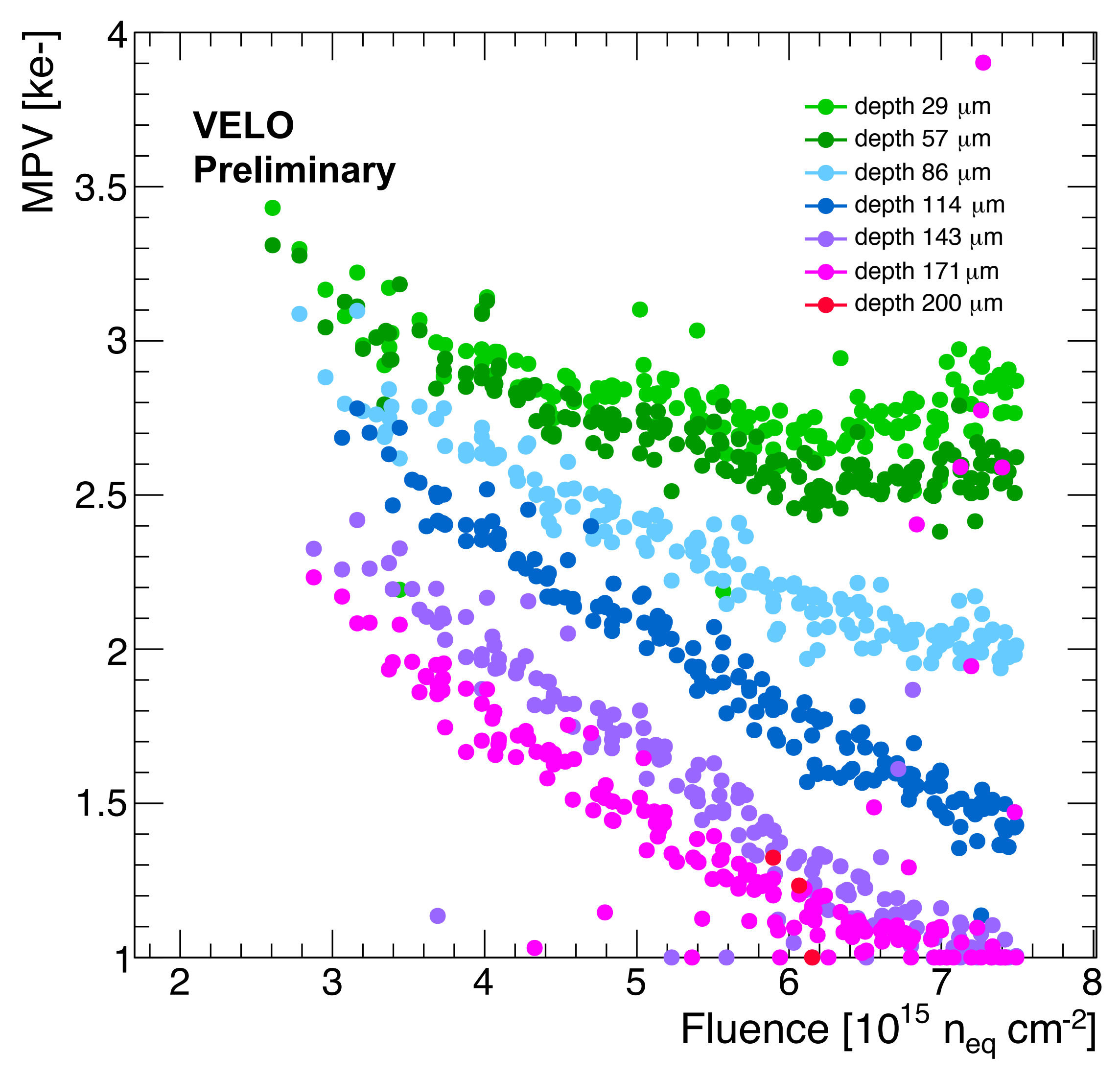

VELO Upgrade

Prototype Sensors

Timepix3 Telescope

- Time Resolution

- Pointing Resolution

Rate Performance

- Sensor Characterisation

- HV Tolerance

Charge Collection

Efficiency

Spatial Resolution

Edge

Grazing Angles

- Summary

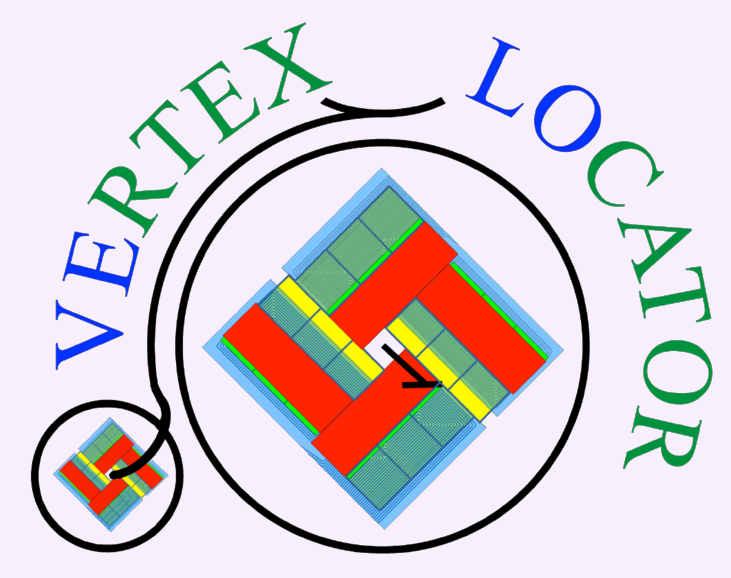


- Timepix3 telescope has outstanding performance

- Allowed to characterise a large variety of prototype sensors for the LHCb VELO upgrade

- 400 sensors already delivered and under test

- First VELO upgrade modules are being constructed

VELO modules tested in beam a couple of weeks ago using the Timepix3 telescope!

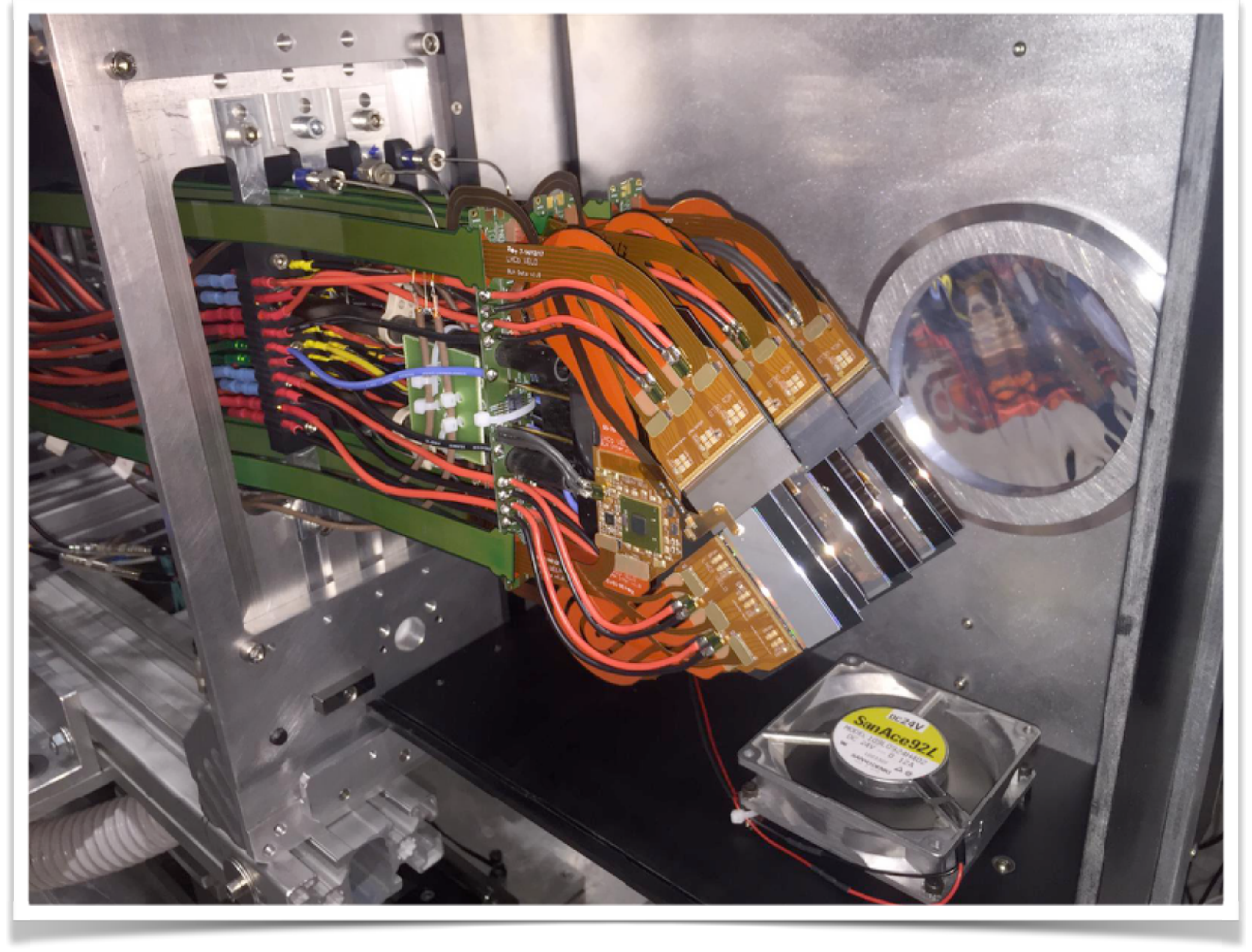

- VELO Upgrade

- Prototype Sensors

- Timepix3 Telescope

- Time Resolution

- Pointing Resolution

Rate Performance

- Sensor Characterisation - HV Tolerance

- Charge Collection

- Efficiency

- Spatial Resolution

- Edge

- Grazing Angles

- Summary

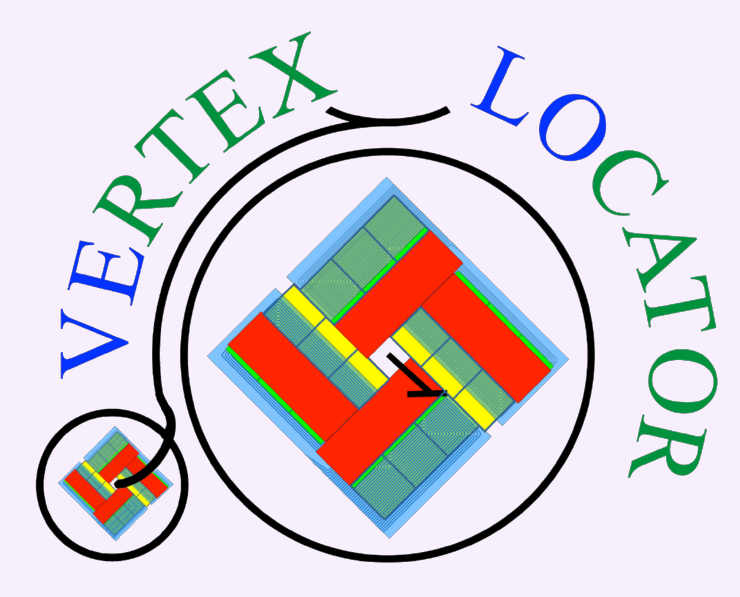


Back Up 
The sensor is rotated at a very large angle (83-89 deg) with respect to the beam

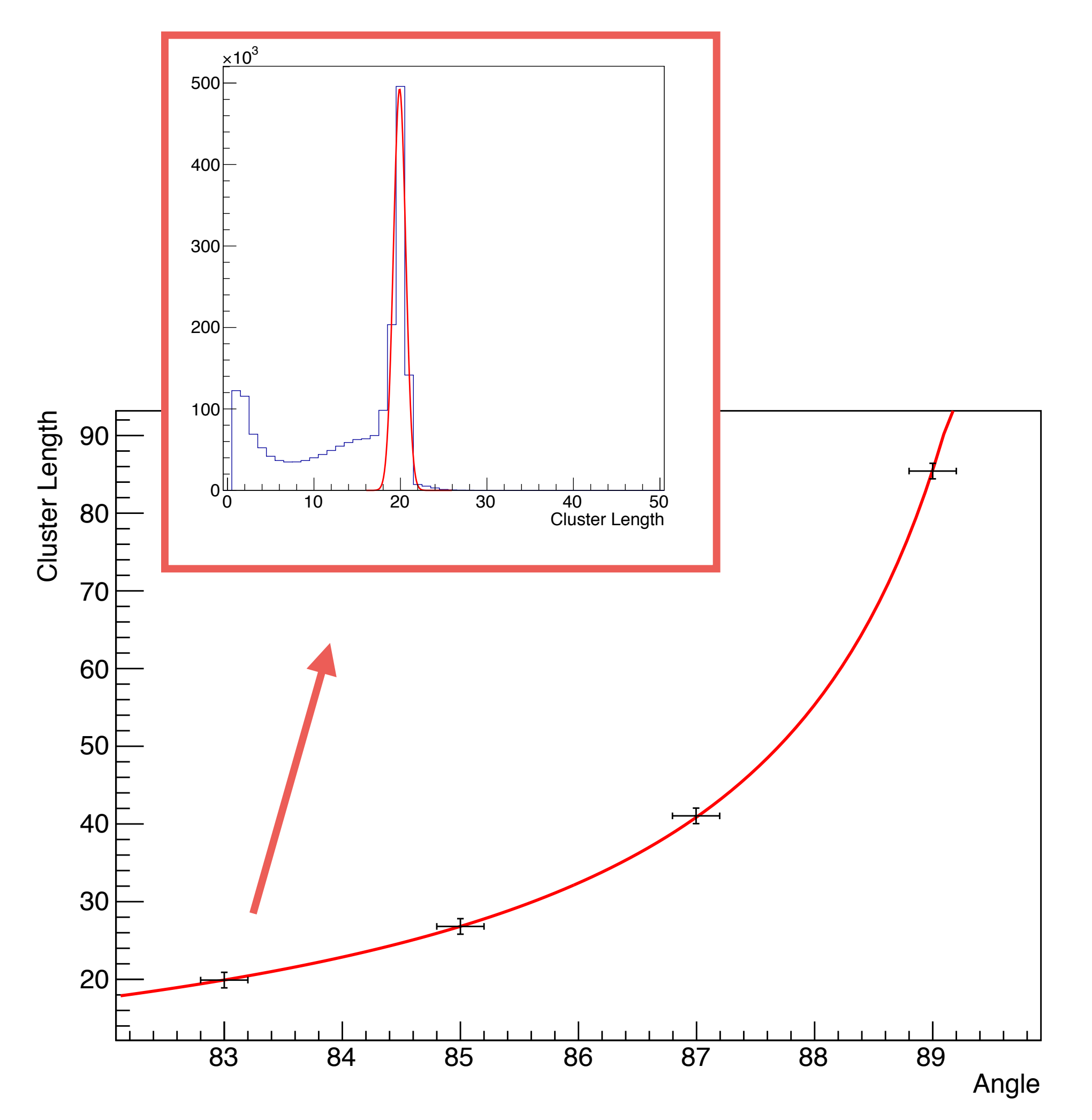

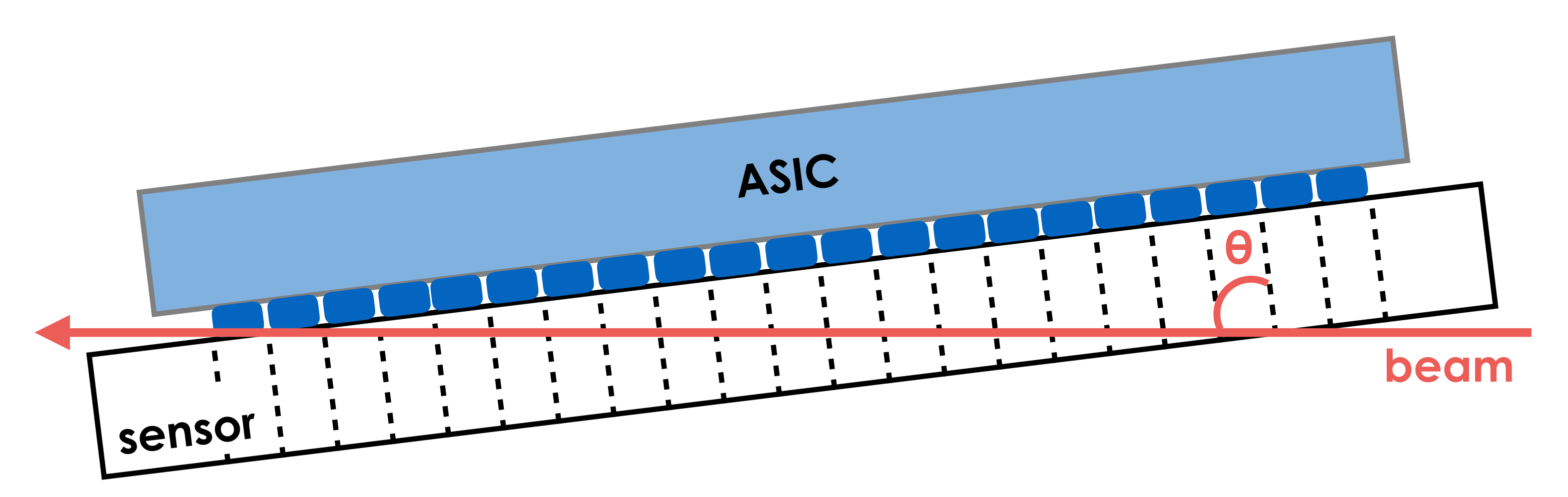

- long tracks through multiple adjacent pixels

- The number of columns traversed by the track depends on the angle

- a fit of the nominal cluster length as a function of the angle allows to extract the effective depletion depth!
VELO Upgrade

- Prototype Sensors

Timepix3 Telescope

- Time Resolution

Pointing Resolution

Rate Performance

- Sensor Characterisation

- HV Tolerance

Charge Collection

Efficiency

Spatial Resolution

Edge

- Grazing Angles

summary

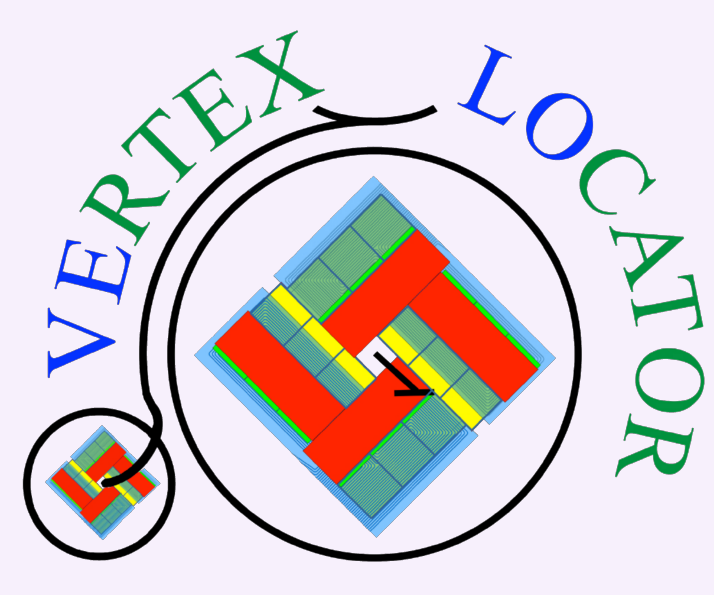



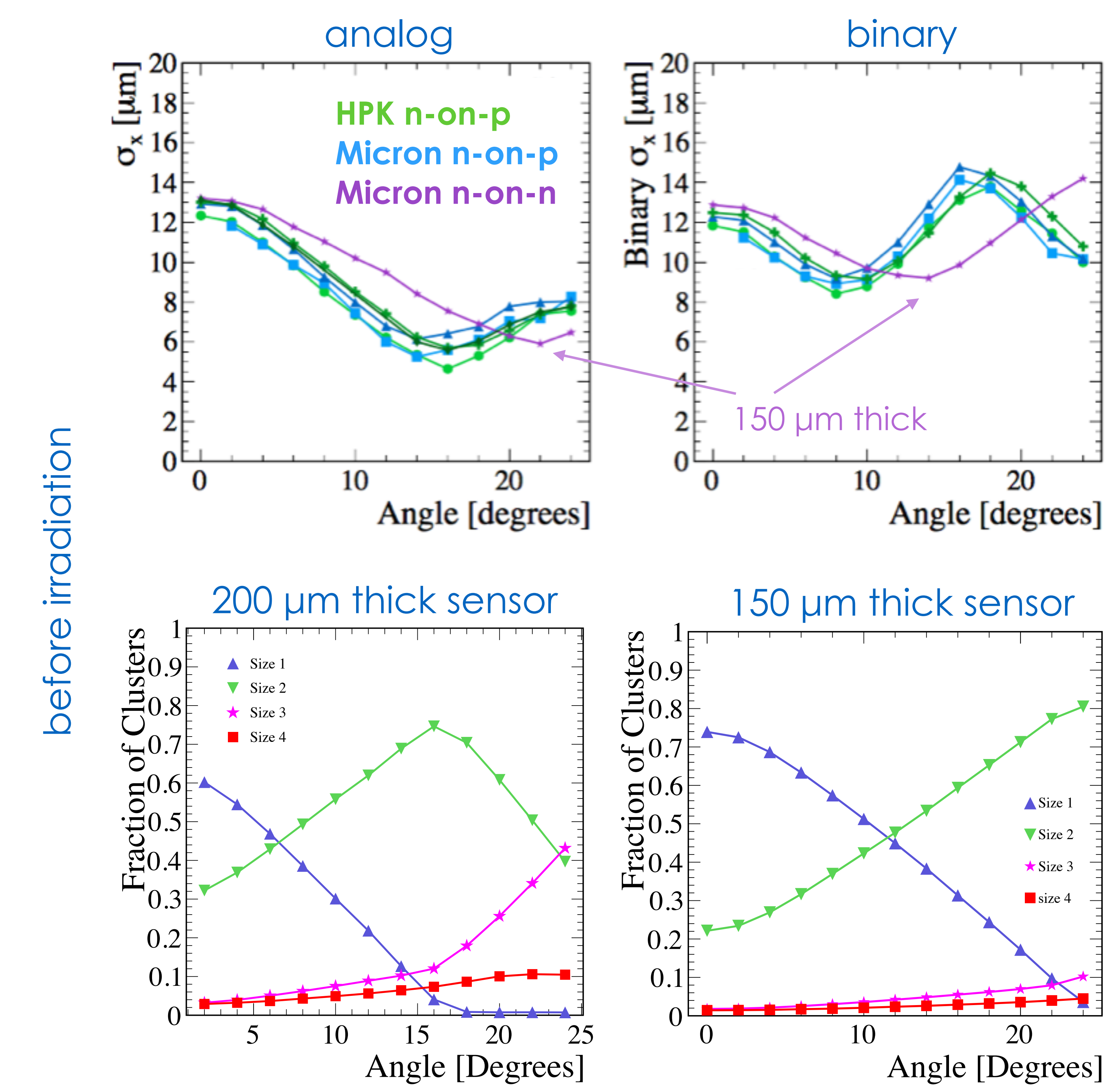

spatial resolution as a function of angle

fractions clusters as a function of angle
VELO Upgrade

Prototype Sensors

Timepix3 Telescope

- Time Resolution

Pointing Resolution

Rate Performance

- Sensor Characterisation

HV Tolerance

Charge Collection

Efficiency

Spatial Resolution

Edge

Grazing Angles

- Summary

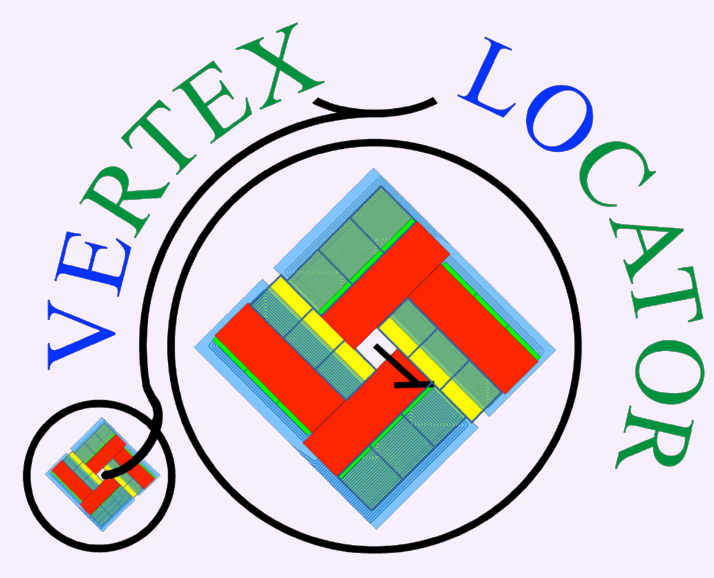


